REPRESENTATION THEORY

An Electronic Journal of the American Mathematical Society

Volume 15, Pages 531-567 (July 7, 2011)

S 1088-4165(2011)00403-9

\title{
ON THE COMPUTABILITY OF SOME POSITIVE-DEPTH SUPERCUSPIDAL CHARACTERS NEAR THE IDENTITY
}

\author{
RAF CLUCKERS, CLIFTON CUNNINGHAM, JULIA GORDON, AND LOREN SPICE
}

\begin{abstract}
This paper is concerned with the values of Harish-Chandra characters of a class of positive-depth, toral, very supercuspidal representations of $p$-adic symplectic and special orthogonal groups, near the identity element. We declare two representations equivalent if their characters coincide on a specific neighbourhood of the identity (which is larger than the neighbourhood on which the Harish-Chandra local character expansion holds). We construct a parameter space $B$ (that depends on the group and a real number $r>0$ ) for the set of equivalence classes of the representations of minimal depth $r$ satisfying some additional assumptions. This parameter space is essentially a geometric object defined over $\mathbb{Q}$. Given a non-Archimedean local field $\mathbb{K}$ with sufficiently large residual characteristic, the part of the character table near the identity element for $G(\mathbb{K})$ that comes from our class of representations is parameterized by the residue-field points of $B$. The character values themselves can be recovered by specialization from a constructible motivic exponential function, in the terminology of Cluckers and Loeser in a recent paper. The values of such functions are algorithmically computable. It is in this sense that we show that a large part of the character table of the group $G(\mathbb{K})$ is computable.
\end{abstract}

\section{INTRODUCTION}

Recent years have seen considerable progress in the program of studying harmonic analysis on $p$-adic groups in a field-independent fashion. This paper takes another step in that program.

On one hand, we are motivated by the hope of an analogy with the case of finite groups of Lie type, where for many representations, character values "near the identity" are given by Green polynomials, and where in general the characters are recovered from geometric objects. On the other hand, we are guided by the examples in [23, 27] that indicate that for a representation of a $p$-adic group, the character values at certain families of topologically unipotent, regular semisimple elements can be expressed in terms of the numbers of rational points of a family of hyperelliptic curves over the residue field.

We consider in this paper a class of functions that includes both rational functions in the cardinality of the residue field and functions defined by such geometric counting methods; namely, the class of constructible exponential functions defined in [10. See 2.7 for details. The key feature of these functions is that they can be

Received by the editors April 19, 2009 and, in revised form, January 29, 2010 and February 4, 2011.

2010 Mathematics Subject Classification. Primary 22E50, 03C98.

Key words and phrases. Character, orbital integral, motivic integration, supercuspidal representation. 
described in a field-independent manner, by means of a formal language of logic. Our hope is that Harish-Chandra character of a supercuspidal representation of a $p$-adic group belongs to this class of functions. In this paper, we prove that this is the case for the restrictions to a neighbourhood of the identity of the characters of a certain class of toral, very supercuspidal, positive-depth representations of symplectic and (split) special orthogonal groups. A similar result (stated in slightly different terms) was proved in 17 for a class of supercuspidal, depth-zero representations (the so-called Deligne-Lusztig representations) of symplectic and odd orthogonal groups.

Following the program outlined in [21, we will prove our result by using the theory of motivic integration and the language of definable subassignments. The specific version of motivic integration that we use here was developed by R. Cluckers and F. Loeser in [10,11. We refer to these original papers for details, and to the expository articles [9, 19, 22, for an introduction to these ideas and the language.

When we try to talk about the Harish-Chandra characters of the representations of a $p$-adic matrix group in a field-independent way, we encounter the issue of being unable to identify how we can keep the representation the "same" while varying the ground field. More precisely, at present, we do not know if the construction of supercuspidal representations can be carried out within the formal language that we use, without fixing the field first; so any question about "the character of a given representation $\pi$ " is not well defined in this context. Instead, we construct, for each local, non-Archimedean field $\mathbb{K}$, a space, over the residue field of $\mathbb{K}$, whose rational points parameterize $r$-equivalence classes of supercuspidal representations, in the sense of Definition 2.9. See Definition 4.4. The necessity of constructing this parameter space before even beginning to speak of character values requires us to impose numerous conditions on the representations that we consider.

Once we construct the parameter space, we show that there is a constructible motivic exponential function (whose variables are the parameters for the representation, and a group element) whose specialization to $\mathbb{K}$ encodes the restrictions of the Harish-Chandra characters of the representations of $G(\mathbb{K})$ that we consider, up to a rational constant. See Theorem 2.23 ,

Now we say a few words about the methods of this construction, and the assumptions that, at present, we have to impose on the representations that we consider. Our main strategy is to express the restriction of the character to a neighbourhood of the identity as a Fourier transform of an orbital integral at a semisimple element, and then to use the results of 14 about good orbital integrals.

To make this more precise, for now, let $\mathbb{K}$ be a fixed local, non-Archimedean field, and $G$ a connected, reductive, algebraic group defined over $\mathbb{K}$. In 28, Kim and Murnaghan describe the theory of $\Gamma$-asymptotic expansions. This is a generalization of Murnaghan-Kirillov theory (see [4,35]) that associates to a smooth, irreducible representation $\pi$ of $G(\mathbb{K})$ satisfying certain conditions (see [28, Definition 4.1.3(2)]) a $\mathbb{K}$-rational, semisimple element $\Gamma$ in the Lie algebra of $G(\mathbb{K})$ (see Theorem 4.4.1 loc. cit.). In order to combine these results with those of Cunningham-Hales 14, we need to impose restrictions on $\Gamma$ (see Definition 2.3). These conditions imply that the representation $\pi$ is supercuspidal (indeed, toral and "very supercuspidal"), so that we may use the explicit construction described by J.-K. Yu (see [44] and our $§ 2.6$ ). 
Once we have constructed the parameter space for the good orbital integrals that arise from the characters that we consider, we use motivic integration to show that the distribution characters, evaluated at families of constructible test functions supported on the given neighbourhood of the identity element, again produce constructible functions in the parameters. From this, we proceed to show that the Harish-Chandra character function is, in fact, a constructible exponential function, using a result of J. Korman on the local constancy of characters 31] (which we reproduce here as Theorem 4.8). This is carried out in Section 4.4. We observe that the local constancy result by itself is not sufficient to conclude that the character is a constructible exponential function, and we need all our conclusions about the distribution character in addition to it. In Section 3, we offer a more general perspective on distributions and definability, which we expect to be useful in the context of motivic integration.

To summarize, starting with a symplectic or special orthogonal group $G$, and a positive real number $r$, we produce a definable subassignment $B_{\mathfrak{g},-r}$ whose $\mathbb{K}_{\mathbb{K}^{-}}$ points parameterize the $r$-equivalence classes of "restricted" representations (in the sense defined in Section 2.6) of $G(\mathbb{K})$ of minimal depth $r$, where $\mathbb{K}_{\mathbb{K}}$ stands for the residue field of $\mathbb{K}$. We then define, for each $y \in B_{\mathfrak{g},-r}\left(\mathbb{k}_{\mathbb{K}}\right)$, a constructible exponential motivic function on the "subassignment" of regular topologically unipotent elements in $G$ such that the restriction of its specialization to a neighbourhood of the identity (depending on $r$ ) coincides with the restrictions of the Harish-Chandra characters of the representations of $G(\mathbb{K})$ in the $r$-equivalence class that corresponds to the point $y$.

By definition, the values of such constructible exponential functions are, in principle, algorithmically computable. It is in this sense that we show that, in principle, there exists an algorithm that generates the part of the character table of $G$ that corresponds to the character values of restricted representations of a given minimal depth, in a neighbourhood of the identity element (which depends on the depth).

Acknowledgements. The third-named author is grateful to Fiona Murnaghan for helpful conversations and encouragement, and to Max Planck Institute in Bonn for its hospitality while a part of this paper was written. The fourth-named author was supported by NSF Postdoctoral Fellowship DMS-0503108.

\section{Preliminaries}

In order to apply the results of [14, we assume throughout that $G$ is a symplectic group $G=\mathrm{Sp}_{2 N}$ or a split special orthogonal group $G=\mathrm{SO}_{n}$. In the first case, we write $n_{G}=2 N$. In the second, we write $n_{G}=n$. Thus, $n_{G}$ should be thought of as the "natural matrix size" of elements of $G$. We will denote the Lie algebra of $G$ by $\mathfrak{g}$, and the linear dual of $\mathfrak{g}$ by $\mathfrak{g}^{*}$.

The symbol $\mathbb{K}$ is reserved for a non-Archimedean, local field (with no assumption on the characteristic); i.e., a finite extension of $\mathbb{Q}_{p}$, or the field of Laurent series over a finite field. We will henceforth usually drop the adjective "non-Archimedean". We will assume whenever convenient that the residual characteristic, $p$, of $\mathbb{K}$ is larger than some specified bound. In particular, we shall always assume that it is odd.

The ring of integers of $\mathbb{K}$ will always be denoted by $\mathcal{O}_{\mathbb{K}}$, and the residue field of $\mathbb{K}$ by $\mathbb{k}_{\mathbb{K}}$. We write $\mathbb{K}^{\text {unr }}$ (respectively, $\mathbb{K}^{\text {tame }}$ ) for an arbitrary unramified (respectively, tame) closure of $\mathbb{K}$. If $E / \mathbb{K}$ is an algebraic extension, then there is a unique 
valuation, $\operatorname{ord}_{\mathbb{K}}$, on $E$ such that $\operatorname{ord}_{\mathbb{K}}(\mathbb{K})=\mathbb{Z}$. We call $\operatorname{ord}_{\mathbb{K}}$ the $\mathbb{K}$-normalised valuation (on $E$ ).

In all cases, we realize $G$ explicitly as the group $\left\{X \in \mathrm{GL}_{n} \mid{ }^{\mathrm{t}} X J X=J\right\}$, where $J$ is the matrix satisfying

$$
J_{i j}= \begin{cases}(-1)^{i}, & i+j=n_{G}+1, \\ 0, & \text { otherwise }\end{cases}
$$

in the symplectic case; and

$$
J_{i j}= \begin{cases}1, & i+j=n_{G}+1, \\ 0, & \text { otherwise, }\end{cases}
$$

in the special orthogonal case. Note that, with this definition, $G$ is automatically $\mathbb{Z}$-split in the even special orthogonal case.

Recall that there is associated to $G$ (or any reductive group over $\mathbb{K}$ ) a polysimplicial $G(\mathbb{K})$-set $\mathcal{B}(G, \mathbb{K}$ ), the building of $G$ over $\mathbb{K}$ (see [7, §7.4], where $\mathcal{I}$ is used instead), and, for each $x \in \mathcal{B}(G, \mathbb{K})$, a compact, open subgroup $G(\mathbb{K})_{x}$ of $G(\mathbb{K})$, called a parahoric subgroup (see [8, Proposition 4.6.28(i)], where $P_{\{x\}}^{0}$ is used instead). Usually one would need to distinguish between the reduced and enlarged buildings, but, in our situation, $G$ is semisimple, so there is no difference.

Moy and Prasad have described, for each point $x \in \mathcal{B}(G, \mathbb{K})$, filtrations:

- of $\mathfrak{g}(\mathbb{K})$ by compact, open lattices $\mathfrak{g}(\mathbb{K})_{x, r}$,

- of $\mathfrak{g}^{*}(\mathbb{K})$ by compact, open lattices $\mathfrak{g}^{*}(\mathbb{K})_{x, r}$, and

- of $G(\mathbb{K})_{x}$ by compact, open, normal subgroups $G(\mathbb{K})_{x, r}$ with $G(\mathbb{K})_{x}=$ $G(\mathbb{K})_{x, 0}$

(see [33, $\S \S 2.6,3.2-3.3]$, where $P_{x, r}$ is used instead of $G(\mathbb{K})_{x, r}$, and [34, §3.2-3.3], where $\mathcal{G}_{x, r}$ is used instead of $G(\mathbb{K})_{x, r}$ ). The indexings of these filtrations depend on the choice of normalization of valuation. The index $r$ usually ranges over $\widetilde{\mathbb{R}} \backslash\{\infty\}$ (where the set $\widetilde{\mathbb{R}}$ is as in 7 , $\S 6.4 .1]$ ), but we will find it convenient to put $\mathfrak{g}(\mathbb{K})_{x, \infty}=$ $\{0\}, \mathfrak{g}^{*}(\mathbb{K})_{x, \infty}=\{0\}$, and $G(\mathbb{K})_{x, \infty}=\{1\}$ for all $x \in \mathcal{B}(G, \mathbb{K})$. These satisfy the obvious $G(\mathbb{K})$-equivariance properties, such as $\operatorname{Int}(g) G(\mathbb{K})_{x, r}=G(\mathbb{K})_{g \cdot x, r}$ for all $g \in G(\mathbb{K}), x \in \mathcal{B}(G, \mathbb{K})$, and $r \in \widetilde{\mathbb{R}}$, where Int is the action of $G$ on itself by conjugation.

Remark 1.1. In our case, if we realize $G$ as the fixed-point group of a $\mathbb{K}$-involution $\sigma$ on $\mathrm{SL}_{n}$, then there is a natural involution $\mathcal{B}(\sigma)$ on $\mathcal{B}\left(\mathrm{SL}_{n}, \mathbb{K}\right)=\mathcal{B}^{\text {red }}\left(\mathrm{GL}_{n}, \mathbb{K}\right)$, the reduced building of $\mathrm{GL}_{n}$ over $\mathbb{K}$, such that $\mathcal{B}(G, \mathbb{K})$ may be identified (as a metric space) with the $\mathcal{B}(\sigma)$-fixed points in $\mathcal{B}^{\text {red }}\left(\mathrm{GL}_{n}, \mathbb{K}\right)$. See 30. Theorem 6.7.3]. Further, if $x \in \mathcal{B}(G, \mathbb{K})$ and $t \in \widetilde{\mathbb{R}}_{>0}$, then $G(\mathbb{K})_{x, t}=G(\mathbb{K}) \cap \mathrm{GL}_{n}(\mathbb{K})_{x, t}$.

If $t \in \widetilde{\mathbb{R}}$, then we write $\mathfrak{g}(\mathbb{K})_{t}=\bigcup_{x \in \mathcal{B}(G, \mathbb{K})} \mathfrak{g}(\mathbb{K})_{x, t}, \mathfrak{g}^{*}(\mathbb{K})_{t}=\bigcup_{x \in \mathcal{B}(G, \mathbb{K})} \mathfrak{g}^{*}(\mathbb{K})_{x, t}$, and, if $t \geq 0, G(\mathbb{K})_{t}=\bigcup_{x \in \mathcal{B}(G, \mathbb{K})} G(\mathbb{K})_{x, t}$. If $u \in \widetilde{\mathbb{R}}$ with $t \leq u$, then we write $\mathfrak{g}(\mathbb{K})_{x, t: u}=\mathfrak{g}(\mathbb{K})_{x, t} / \mathfrak{g}(\mathbb{K})_{x, u}, \mathfrak{g}^{*}(\mathbb{K})_{x, t: u}=\mathfrak{g}^{*}(\mathbb{K})_{x, t} / \mathfrak{g}^{*}(\mathbb{K})_{x, u}$, and, if $t \geq 0$, $G(\mathbb{K})_{x, t: u}=G(\mathbb{K})_{x, t} / G(\mathbb{K})_{x, u}$.

If $X \in \mathfrak{g}(\mathbb{K}), g \in G(\mathbb{K})$, and $x \in \mathcal{B}(G, \mathbb{K})$, then we write

- $\mathrm{d}_{x}(X)=t$ if $t \in \mathbb{R}$ satisfies $X \in \mathfrak{g}(\mathbb{K})_{x, t} \backslash \mathfrak{g}(\mathbb{K})_{x, t^{+}}$, and $\mathrm{d}_{x}(X)=\infty$ if $X=0$

- $\mathrm{d}(X)=\sup \left\{\mathrm{d}_{x}(X) \mid x \in \mathcal{B}(G, \mathbb{K})\right\}$; 
- $\mathrm{d}_{x}(g)=t$ if $t \in \mathbb{R}_{\geq 0}$ satisfies $g \in G(\mathbb{K})_{x, t} \backslash G(\mathbb{K})_{x, t^{+}}$, and $\mathrm{d}_{x}(g)=\infty$ if $g=1 ;$ and

- $\mathrm{d}(g)=\sup \left\{\mathrm{d}_{x}(g) \mid x \in \mathcal{B}(G, \mathbb{K}), g \in G(\mathbb{K})_{x}\right\}$.

Notice that we do not define $\mathrm{d}_{x}(g)$ if $g \notin G(\mathbb{K})_{x, 0}$, and we do not define $\mathrm{d}(g)$ if $g \notin G(\mathbb{K})_{0}$. We call $\mathrm{d}(X)$ (respectively, $\left.\mathrm{d}_{x}(X)\right)$ the depth (respectively, $x$-depth) of $X$, and similarly for group elements.

Moy and Prasad also define analogues of these objects when the ground field is changed from $\mathbb{K}$ to an algebraic extension with finite ramification degree (for example, $\left.\mathbb{K}^{\text {unr }}\right)$; we will use the obvious notation for these objects.

By definition, an element $g \in G(\mathbb{K})$ is compact if the closed subgroup that it generates is compact. This is equivalent to its orbits in $\mathcal{B}(G, \mathbb{K})$ being bounded [7. (4.4.9)], hence, by [7, Proposition 3.2.4], to $g$ possessing a fixed point in $\mathcal{B}(G, \mathbb{K})$.

Let e be the Cayley transform $X \mapsto(1+X)(1-X)^{-1}$, which identifies the topologically nilpotent set in $\mathfrak{g}\left(\mathbb{K}^{\text {unr }}\right)$ with the topologically unipotent set in $G\left(\mathbb{K}^{\text {unr }}\right)$. We have that

- the family of restrictions of e to the filtration lattices $\mathfrak{g}\left(\mathbb{K}^{\text {unr }}\right)_{x, 0^{+}}$satisfy 4. Property (CE1)], so that, for every $x \in \mathcal{B}\left(G, \mathbb{K}^{\text {unr }}\right)$ and pair $t, u \in \widetilde{\mathbb{R}}_{>0}$ with $2 t \geq u$, we have that e induces an isomorphism $\mathrm{e}_{x, t: u}: \mathfrak{g}\left(\mathbb{K}^{\mathrm{unr}}\right)_{x, t: u} \rightarrow$ $G\left(\mathbb{K}^{\mathrm{unr}}\right)_{x, t: u}$;

- the resulting isomorphisms satisfy the equivariance property

$$
\mathrm{e}_{g \cdot x, t: u} \circ \operatorname{Ad}(g)=\operatorname{Int}(g) \circ \mathrm{e}_{x, t: u}
$$

for all $x, t$, and $u$ as above, and all $g \in G\left(\mathbb{K}^{\text {unr }}\right.$ ) (here Ad is the adjoint action of $G$ on $\mathfrak{g}$ ); and

- if $T$ is a maximal $\mathbb{K}^{\text {unr }}$-torus in $G, \alpha$ is an absolute root of $T$ in $G$ with differential $d \alpha, t \in \mathbb{R}_{>0}, X \in \mathfrak{t}\left(\mathbb{K}^{\text {unr }}\right)_{0^{+}}$, and $\operatorname{ord}(d \alpha(X))=t$, then

$$
\operatorname{ord}_{\mathbb{K}}(d \alpha(X)-(\alpha(\mathrm{e}(X))-1))>t .
$$

With the notation of Remark 1.1 we have that $\mathrm{e}(X) \equiv 1+2 X\left(\bmod \mathrm{GL}_{n}(E)_{x, 2 t}\right)$ whenever $x \in \mathcal{B}(G, E), t \in \widetilde{\mathbb{R}}_{>0}$, and $X \in \mathfrak{g}(E)_{x, t}$. When the residual characteristic of $\mathbb{K}$ is odd, the first and third facts follow from easily verified facts about the behaviour of the map $X \mapsto 1+X$, since $\operatorname{GL}_{n}(E)_{x, 2 t} \cap G(E)=G(E)_{x, 2 t}$. The second fact is obvious from the definition.

We will usually be concerned only with the behaviour of these maps on $\mathbb{K}$-rational points, but the fact that we can work with them over $\mathbb{K}^{\text {unr }}$ comes in handy when we discuss stable conjugacy.

\section{Restricted REPRESEntations}

In this section, we define the class of representations of $G(\mathbb{K})$ that we will consider.

2.1. Restricted elements. We recall a notion introduced in [1, Definition 2.2.4].

Definition 2.1. If $G$ is any connected, reductive group defined over a local field $\mathbb{K}$, and $\mathfrak{g}$ is the Lie algebra of $G$, then an element $X \in \mathfrak{g}(\mathbb{K})$ (respectively, $X^{*} \in \mathfrak{g}^{*}(\mathbb{K})$ ) is good of depth $r$ if

(1) there is a maximal $\mathbb{K}$-torus $T$ in $G$, with Lie algebra $\mathfrak{t}$, that splits over $\mathbb{K}^{\text {tame }}$

(2) $X \in \mathfrak{t}(\mathbb{K})_{r} \backslash \mathfrak{t}(\mathbb{K})_{r^{+}}$(respectively, $\left.X^{*} \in \mathfrak{t}^{*}(\mathbb{K})_{r} \backslash \mathfrak{t}^{*}(\mathbb{K})_{r^{+}}\right)$; and 
(3) for each (absolute) root (respectively, coroot) $\alpha$ of $T$ in $G, d \alpha(X)$ is 0 , or has $\mathbb{K}$-normalised valuation $r$.

Remark 2.2. Preserve the notation of Definition 2.1. By [3, Corollary 3.5.6] (or its obvious analogue for the dual Lie algebra), Definition 2.1(2) implies that $r$ is the depth of $X$ in $\mathfrak{g}(\mathbb{K})$ (respectively, of $X^{*}$ in $\mathfrak{g}^{*}(\mathbb{K})$ ), thus justifying the terminology. For the groups $G$ that we consider, the indices in the character lattice of $T$ of the root lattice of $T$ in $G$, and in the cocharacter lattice of $T$ of the coroot lattice of $T$ in $G$, divide 4 . Recall that $p \neq 2$. Thus, by the definition of the filtration on $\mathfrak{t}(\mathbb{K}$ ) (see [1. p. 9]), if $X$ (respectively, $X^{*}$ ) satisfies Definition 2.1(3), then it automatically lies in $\mathfrak{t}(\mathbb{K})_{r}$ (respectively, $\left.\mathfrak{t}^{*}(\mathbb{K})_{r}\right)$; and, in fact, it equals 0 or satisfies Definition 2.1(2).

The following notion was introduced in [14, Definition 2.4], where the term "slope" was used in place of "depth".

Definition 2.3. Let $r$ be a rational number and $\mathfrak{g}$ a classical Lie algebra defined over $\mathbb{K}$. An element $X$ of $\mathfrak{g}(\mathbb{K})$ is restricted of depth $r$ in $\mathfrak{g}(\mathbb{K})$ if it satisfies the following conditions:

(1) it is good of depth $r$ (in the sense of Definition 2.1);

(2) it is regular;

(3) each eigenvalue is 0 , or has $\mathbb{K}$-normalised valuation $r$; and

(4) the multiplicity of the eigenvalue 0 is at most 1.

The set of elements of $\mathfrak{g}(\mathbb{K})$ that are restricted of depth $r$ is denoted by $\mathfrak{g}(r, \mathbb{K})$.

Remark 2.4. Note that restricted elements are, a fortiori, regular semisimple. Preserve the notation of Definition 2.3 .

- For $\mathfrak{g}$ a symplectic Lie algebra, if $\lambda$ is an eigenvalue of $X$, then $2 \lambda$ is a root value for $X$. Accordingly, in odd characteristic, Definition 2.3)(2) implies Definition 2.3(4); and Definition 2.3(3) implies that if $X \neq 0$ is good of depth $r$, then it is restricted of depth $r$.

- For $\mathfrak{g}$ a special orthogonal Lie algebra (even or odd), if 0 is an eigenvalue of $X$ with multiplicity at least 2, then it is also a root value of $X$. Accordingly, Definition 2.3(2) implies Definition 2.3(4).

- For $\mathfrak{g}$ an odd orthogonal Lie algebra, if $\lambda$ is a non-zero eigenvalue of $X$, then it is also a root value of $X$. Accordingly, Definition 2.3(11) implies Definition 2.3(3).

That is, an element of a symplectic or odd special orthogonal Lie algebra is restricted of depth $r$ if and only if it is regular, and good of depth $r$. This implication fails for even special orthogonal Lie algebras.

For a given classical Lie algebra $\mathfrak{g}$, the set of $r \in \mathbb{Q}$ for which $\mathfrak{g}(r, \mathbb{K})$ is non-empty is independent of $\mathbb{K}$, for sufficiently large residual characteristic.

2.2. Thickened orbits. We recall [14, Definition 2.5], which introduces an equivalence relation on the class of restricted elements of fixed depth in a Lie algebra.

If $\mathfrak{g}$ is a symplectic or odd special orthogonal Lie algebra, then two restricted elements $X$ and $X^{\prime}$ of depth $r$ are $r$-equivalent if the multi-sets of eigenvalues $\left\{\lambda_{i} \mid 1 \leq i \leq n_{G}\right\}$ of $X$ and $\left\{\lambda_{i}^{\prime} \mid 1 \leq i \leq n_{G}\right\}$ of $X^{\prime}$ can be indexed so that

$$
\forall 1 \leq i \leq n_{G}, \quad \operatorname{ord}_{\mathbb{K}}\left(\lambda_{i}^{\prime}-\lambda_{i}\right)>r .
$$


If $\mathfrak{g}$ is an even special orthogonal Lie algebra $\mathfrak{s o}(2 N)$ (so that $n_{G}=2 N$, in the notation of (1), then we say that $X, X^{\prime} \in \mathfrak{g}(r, \mathbb{K})$ are $r$-equivalent if $(*)$ holds, and, in addition,

$$
\operatorname{ord}_{\mathbb{K}}\left(\operatorname{pfaff}(J X)-\operatorname{pfaff}\left(J X^{\prime}\right)\right)>N r,
$$

where $J$ is as in (2) and, for a skew-symmetric matrix $Y$, $\operatorname{pfaff}(Y)$ is the Pfaffian of $Y$.

In all cases, when $X$ is an element of $\mathfrak{g}(r, \mathbb{K})$, the $r$-equivalence class of $X$ is denoted by $[X]_{r}$.

The above definition is somewhat ad hoc and particular to our situation. We present below an equivalent definition that will generalize readily to other Lie algebras.

Definition 2.5. Suppose $X \in \mathfrak{g}(\mathbb{K})$ is regular semisimple and the depth of $X$ is $r$.

(1) We define

$$
\mathcal{O}_{r}(X):=\bigcup_{Y \in \mathfrak{t}(\mathbb{K})_{r+}} \mathcal{O}(X+Y)
$$

where $\mathfrak{t}(\mathbb{K})$ is the Cartan subalgebra containing $X$ and where $\mathcal{O}(X+Y)$ is the $G(\mathbb{K})$-adjoint orbit of $X+Y$ in $\mathfrak{g}(\mathbb{K})$. We refer to $\mathcal{O}_{r}(X)$ as the thickened orbit of $X$.

(2) We define

$$
\mathcal{O}_{r}^{\text {st }}(X):=\bigcup_{Y \in \mathfrak{t}(\mathbb{K})_{r+}} \mathcal{O}^{\text {st }}(X+Y),
$$

where $\mathfrak{t}(\mathbb{K})$ is the Cartan subalgebra containing $X$. We refer to $\mathcal{O}_{r}^{\text {st }}(X)$ as the stable thickened orbit of $X$.

Lemma 2.6 assures us that the latter notion is the proper way to understand $r$-equivalent elements in $\mathfrak{g}(r, \mathbb{K})$.

Lemma 2.6 ([14, Theorem 4.6]). For each $X \in \mathfrak{g}(r, \mathbb{K}), \mathcal{O}_{r}^{s t}(X)=[X]_{r}$.

Proof. The proof of this lemma is contained in the proof of [14, Theorem 4.6]. We explain this in detail in the proof of Proposition 4.6(1) below, where we actually prove a more precise statement about thickened orbits ( $v s$. stable thickened orbits).

2.3. Thickened orbits and orbital integrals. In 14 it is shown how to recognize the stable thickened orbit $\mathcal{O}_{r}^{\text {st }}(X)=[X]_{r}$ as a $\mathbb{k}_{\mathbb{K}}$-point on a scheme $S_{\mathfrak{g}, r}$. As we will see, one of our main tasks in this paper is to parameterize the thickened orbits of $r$-restricted elements by the residue-field points of an appropriate object. To do that, we will use the parameterization of stable thickened orbits from [14]. In the meantime, the following proposition reveals the relation between thickened orbits of $r$-restricted elements and orbital integrals on $\mathcal{H}_{r}(\mathfrak{g}(\mathbb{K}))$ (defined below).

Definition 2.7. Put

$$
\mathcal{H}_{r}(\mathfrak{g}(\mathbb{K}))=\sum_{x \in \mathcal{B}(G, \mathbb{K})} C_{c}\left(\mathfrak{g}(\mathbb{K}) / \mathfrak{g}(\mathbb{K})_{x, r^{+}}\right) .
$$

That is, $\mathcal{H}_{r}(\mathfrak{g}(\mathbb{K}))$ is the space of functions $f \in C_{c}^{\infty}(\mathfrak{g}(\mathbb{K}))$ for which there are some $s \leq r$ and a finite set $\left\{x_{i} \mid i \in I\right\} \subseteq \mathcal{B}(G, \mathbb{K})$ such that $f=\sum_{i \in I} f_{i}$ where $f_{i} \in C\left(\mathfrak{g}(\mathbb{K})_{x_{i}, s: r^{+}}\right)$. 
Let $X \in \mathfrak{g}(\mathbb{K})$. We denote by $\mu_{X}: C_{c}^{\infty}(\mathfrak{g}(\mathbb{K})) \rightarrow \mathbb{C}$ the orbital integral distribution attached to $X$.

Proposition 2.8. Fix $r \in \mathbb{Q}$. Suppose $X, X^{\prime} \in \mathfrak{g}(r, \mathbb{K})$. Then

$$
\mathcal{O}_{r}(X)=\mathcal{O}_{r}\left(X^{\prime}\right) \Longleftrightarrow \forall f \in \mathcal{H}_{r}(\mathfrak{g}(\mathbb{K})), \mu_{X}(f)=\mu_{X^{\prime}}(f) .
$$

Proof. Suppose $\mu_{X}(f)=\mu_{X^{\prime}}(f)$ for all $f \in \mathcal{H}_{r}(\mathfrak{g}(\mathbb{K}))$. Let $T$ be the centralizer of $X$ in $G$ and set $\mathfrak{t}=\operatorname{Lie} T$. Suppose $x \in \mathcal{B}(G, \mathbb{K})$ lies in the image of $\mathcal{B}(T, \mathbb{K})$. (To make sense of this one must observe that $T$ is tamely ramified, since $X \in \mathfrak{g}(r, \mathbb{K})$.) Let $\mathbf{1}_{X+\mathfrak{g}(\mathbb{K})_{x, r^{+}}}$be the characteristic function of $X+\mathfrak{g}(\mathbb{K})_{x, r^{+}}$. Then $\mathbf{1}_{X+\mathfrak{g}(\mathbb{K})_{x, r}} \in$ $\mathcal{H}_{r}(\mathfrak{g}(\mathbb{K}))$, so

$$
\mu_{X}\left(\mathbf{1}_{X+\mathfrak{g}(\mathbb{K})_{x, r+}}\right)=\mu_{X^{\prime}}\left(\mathbf{1}_{X+\mathfrak{g}(\mathbb{K})_{x, r^{+}}}\right) .
$$

Clearly $\mu_{X}\left(\mathbf{1}_{X+\mathfrak{g}(\mathbb{K})_{x, r}+}\right)$ is non-zero. On the other hand, $\mu_{X^{\prime}}\left(\mathbf{1}_{X+\mathfrak{g}(\mathbb{K})_{x, r^{+}}}\right)$is 0 unless the $G(\mathbb{K})$-adjoint orbit of $X^{\prime}$ intersects $X+\mathfrak{g}(\mathbb{K})_{x, r^{+}}$. By [1, Lemma 2.3.2], this is true if and only if the $G(\mathbb{K})$-adjoint orbit of $X^{\prime}$ intersects $X+\mathfrak{t}(\mathbb{K})_{r^{+}}$, in which case $\mathcal{O}_{r}(X)=\mathcal{O}_{r}\left(X^{\prime}\right)$.

Conversely, suppose $\mathcal{O}_{r}(X)=\mathcal{O}_{r}\left(X^{\prime}\right)$. Because orbital integrals are $G(\mathbb{K})$ invariant, we may assume $X^{\prime} \in X+\mathfrak{t}(\mathbb{K})_{r^{+}}$, where $\mathfrak{t}(\mathbb{K})$ is the Cartan subalgebra of $\mathfrak{g}(\mathbb{K})$ containing $X$, as above. Let $x$ be as above and observe that $\mathfrak{t}(\mathbb{K})_{r^{+}} \subseteq \mathfrak{g}(\mathbb{K})_{x, r^{+}}$. Then the image of $X$ under $\mathfrak{g}(\mathbb{K})_{x, r} \rightarrow \mathfrak{g}(\mathbb{K})_{x, r: r^{+}}$coincides with the image of $X^{\prime}$ under the same function. Arguing as in the proof of [14 Corollary 1.30], it follows from [14, Theorem 1.26] and the fact that $\left|D^{\mathfrak{g}, \mathfrak{l}}(X)\right|=\left|D^{\mathfrak{g}, \mathfrak{l}}\left(X^{\prime}\right)\right|$ for any Levi $\mathbb{K}$-subalgebra $\mathfrak{l}$ of $\mathfrak{g}$ containing $C_{\mathfrak{g}}(X)=C_{\mathfrak{g}}\left(X^{\prime}\right)$ (see [14, (6.1.3)]) that $\mu_{X}(f)=\mu_{X^{\prime}}(f)$ for every $f \in \mathcal{H}_{r}(\mathfrak{g}(\mathbb{K}))$.

2.4. $r$-equivalent representations. In parallel to the definition of equivalence of elements, we have a definition of equivalence of representations.

Recall that Moy and Prasad have defined the depth of a representation $\pi$ of $G(\mathbb{K})$ to be the least index $r$ such that, for some $x \in \mathcal{B}(G, \mathbb{K})$, the space of $G(\mathbb{K})_{x, r^{+-}}$ fixed vectors in $\pi$ is non-zero (see [33, Theorem 5.2]). We write $\mathrm{d}(\pi)$, rather than $\varrho(\pi)$, for this depth.

In this paper, we have occasion to distinguish carefully between the distribution character of a representation of $G(\mathbb{K})$ and its "density" function on $G(\mathbb{K})^{\mathrm{reg}}$. If the field $\mathbb{K}$ has characteristic zero, then it is known (see Harish-Chandra's theorem 24]) that there exists a locally summable density function $\theta_{\pi}$, that is locally constant on $G(\mathbb{K})^{\text {reg }}$, such that

$$
\Theta_{\pi}(f)=\int_{G(\mathbb{K})} \theta_{\pi}(g) f(g) d g
$$

for all test functions $f \in C_{c}^{\infty}(G(\mathbb{K}))$. However, for fields $\mathbb{K}$ of positive characteristic, it is only known that a locally constant function $\theta_{\pi}$ on $G(\mathbb{K})^{\text {reg }}$ exists such that equality (3) holds for all test functions $f$ whose support is contained in $G(\mathbb{K})^{\text {reg }}$, and it is in general not known that this function $\theta_{\pi}$ is locally summable on $G(\mathbb{K})$. See [5. Appendix] for a discussion of this question, and further references.

We will always denote the distribution character by $\Theta_{\pi}$, and the function by $\theta_{\pi}$. By "Harish-Chandra character", or just the word "character" without modification, we will always mean the function $\theta_{\pi}$. Note, however, that for the groups we are considering, and for the fields of positive characteristic, it is only conjectural at this point that $\theta_{\pi}$ contains as much information as the distribution character $\Theta_{\pi}$. 
In particular, there is no proof at present that if two representations of a general $p$-adic group have the same character function $\theta_{\pi}$, then their distribution characters coincide.

Definition 2.9. Let $\pi$ and $\pi^{\prime}$ be two smooth, irreducible representations of $G(\mathbb{K})$, and assume that $\pi$ has depth $r$. We say that $\pi$ and $\pi^{\prime}$ are $r$-equivalent if the restrictions of their characters $\theta_{\pi}$ and $\theta_{\pi^{\prime}}$ to the set of regular, semisimple elements in $G(\mathbb{K})_{r}$ coincide.

While $r$-equivalence in the above sense is obviously weaker than the usual equivalence of representations (in the sense of the existence of an invertible intertwining map), it still preserves some interesting information about a representation. For example, two $r$-equivalent representations have the same local character expansion, hence the same wave-front set (see [26]); in particular, both are, or both are not, generic (see [37. Théorème, p. 161 and Remarque 2, pp. 162-163]), and both have the same formal degree. Since a representation has depth $r$ if and only if there is some $x \in \mathcal{B}(G, \mathbb{K})$ such that the restriction of its character to $G(\mathbb{K})_{x, r^{+}}$is not orthogonal to 1 (for the usual scalar product of functions on a compact group), $r$-equivalent representations have the same depth. There is also known to be interesting arithmetic information encoded in the behaviour at depth $r$ of the character of a depth- $r$, supercuspidal representation of a general linear group; see, for example, [13, Theorem 4.2(d)], [38, Proposition 2.9(2)], and [39, Theorem 2.5]. All this information, too, is preserved by $r$-equivalence.

2.5. The Fourier transform. Let $\Lambda$ be an additive character of $\mathbb{K}$ that is nontrivial on $\mathcal{O}_{\mathbb{K}}$, but trivial on its unique prime ideal. We use $\Lambda$ to define a Fourier transform on the space $C_{c}^{\infty}(\mathfrak{g}(\mathbb{K}))$ of compactly supported, locally constant, complexvalued functions on $\mathfrak{g}(\mathbb{K})$ by putting

$$
\hat{f}\left(X^{*}\right)=\int_{\mathfrak{g}(\mathbb{K})} f(Y) \Lambda\left(\left\langle X^{*}, Y\right\rangle\right) d Y
$$

for $f \in C_{c}^{\infty}(\mathfrak{g}(\mathbb{K}))$ and $X^{*} \in \mathfrak{g}^{*}(\mathbb{K})$, where $\langle\cdot, \cdot\rangle$ is the standard pairing between $\mathfrak{g}^{*}(\mathbb{K})$ and $\mathfrak{g}(\mathbb{K})$.

Lemma 2.10 ([14, Lemma 1.8]). With the choices made above, $\mathcal{H}_{r}(\mathfrak{g}(\mathbb{K})$ ) (see Definition 2.7) is the space of functions $f \in C_{c}^{\infty}(\mathfrak{g}(\mathbb{K}))$ such that the support of $\hat{f}$ is contained in $\mathfrak{g}^{*}(\mathbb{K})_{-r}$.

As mentioned in [6, §1.1], if $X^{*} \in \mathfrak{g}^{*}(\mathbb{K})$ is semisimple (in the appropriate sense), then there is a distribution $\hat{\mu}_{X^{*}}: C_{c}^{\infty}(\mathfrak{g}(\mathbb{K})) \rightarrow \mathbb{C}$ such that, for $f \in C_{c}^{\infty}(\mathfrak{g}(\mathbb{K}))$, $\hat{\mu}_{X^{*}}(f)$ is the integral of $\hat{f}$ along the $G(\mathbb{K})$-orbit of $X^{*}$ with respect to a suitable $G(\mathbb{K})$-invariant measure. By [4, Theorem A.1.2], there exists a locally constant function $F$ on $\mathfrak{g}(\mathbb{K})^{\text {reg }}$ such that $\hat{\mu}_{X^{*}}(f)=\int_{\mathfrak{g}(\mathbb{K})} f(X) F(X) d X$ for all $f \in C_{c}^{\infty}\left(\mathfrak{g}(\mathbb{K})^{\text {reg }}\right)$. We will also write $\hat{\mu}_{X^{*}}$ for the function $F$.

2.6. The representations we consider. Recall that J.-K. Yu's construction of supercuspidal representations of $G(\mathbb{K})$ (see 44) takes as parameters certain quintuples $\Psi=\left(\vec{G}, \vec{\phi}, \vec{r}, x, \pi_{-1}\right)$. Namely, $\vec{G}=\left(G^{0}, \ldots, G^{d}=G\right), \vec{\phi}=\left(\phi_{0}, \ldots, \phi_{d}\right)$, and $\vec{r}=\left(0 \leq r_{0}<\cdots<r_{d-1} \leq r_{d}\right)$, where, for any $0 \leq i<d$,

(1) $G^{i}$ is the centralizer of a $\mathbb{K}$-anisotropic, $\mathbb{K}^{\text {tame }}$-split $\mathbb{K}$-torus in $G$; 
(2) $\phi_{i}$ is a character of $G^{i}(\mathbb{K})$ that is $G^{i+1}(\mathbb{K})$-generic of depth $\mathrm{d}(\phi)=r_{i}$, in the sense of [44, $\S 9]$;

(3) $d \geq 0$, in the case $d>0, r_{0}>0$ and $\phi_{d}=1$ or $r_{d-1}<r_{d}$;

(4) $x$ is a vertex of $\mathcal{B}\left(G^{0}, \mathbb{K}\right)$; and

(5) $\pi_{-1}$ is a depth-zero, supercuspidal representation of $G^{0}(\mathbb{K})$ obtained by compact induction from a representation of the normalizer of $G^{0}(\mathbb{K})_{x}$.

Such a quintuple is called a cuspidal datum (or sometimes a generic datum). The ingredients $(\vec{r}, x)$ in the above datum are redundant (see 44, Theorem 3.1 and Lemma 3.3]), but we find it convenient nonetheless to have them available. We will write $G^{i, \Psi}$ for $G^{i}, \phi_{i, \Psi}$ for $\phi_{i}$, and similar notation for the other ingredients, as necessary. Write $\pi_{\Psi}$ for the representation constructed from $\Psi$ in [44, §4]. Then $\mathrm{d}\left(\pi_{\Psi}\right)=r_{d}$. We will also call $r_{d}$ the depth of the datum $\Psi$, and write $\mathrm{d}(\Psi)=r_{d}$. The representations arising via Yu's construction are now conventionally called tame supercuspidals. By [29, Theorem 19.1], all supercuspidal representations of $G(\mathbb{K})$ are tame, as long as $\mathbb{K}$ has sufficiently large residual characteristic (see $\S 3.4$ loc. cit., especially Remark 3.5 there). We will consider only those tame supercuspidals that are not twists of depth-zero representations (i.e., for which $d>0$ ). In this case, the number $r_{d}$ is less interesting than the number $r_{d-1}$, which is the smallest depth of a twist of $\pi_{\Psi}$ by a character of $G(\mathbb{K})$. We call $r_{d-1}$ the essential depth of $\Psi$ (or $\pi_{\Psi}$ ), and say that $\Psi$ (or $\pi_{\Psi}$ ) has minimal depth $r$ if $r_{d-1}=r_{d}=r$.

Remark 2.11. The depth-zero analogue of our Theorem 2.21(3) was proven in [17] by a different method.

Remark 2.12. We will usually identify $\mathfrak{g}^{i}$ and $\mathfrak{z}\left(\mathfrak{g}^{i}\right)$ with subalgebras of $\mathfrak{g}$, as follows. If $S^{i}$ is a torus such that $G^{i}=C_{G}\left(S^{i}\right)$, then we regard $\mathfrak{g}^{i}$ as the algebra of fixed points for the adjoint action of $S^{i}$, and $\mathfrak{z}\left(\mathfrak{g}^{i}\right)$ as the algebra of fixed points for the adjoint action of $G^{i}$. We regard $\mathfrak{g}^{i *}$ and $\mathfrak{z}\left(\mathfrak{g}^{i}\right)^{*}$ as subspaces of $\mathfrak{g}^{*}$ in a similar fashion. We will denote these subgroups, respectively, subspaces, by $G^{i, \Psi}$, respectively, $\mathfrak{g}^{i, \Psi}$ when we want to emphasize that they are part of the datum $\Psi$.

Remark 2.13. If $d=1$ and $G^{0}$ is an (elliptic) torus, then $\pi_{-1}$ is a (linear) character. We may, and do, assume, upon replacing $\phi_{0}$ by $\pi_{-1} \otimes \phi_{0}$, that it is the trivial character. We will then call the pair $\left(G^{0}, \phi_{0}\right)$ a toral, very supercuspidal datum, and write just $\pi=\pi_{\left(G^{0}, \phi_{0}\right)}$ for the associated representation.

For $0 \leq i<d$, we can associate to the character $\phi_{i}$ of $G^{i}(\mathbb{K})$ an element $X_{i}^{*}=$ $X_{i, \Psi}^{*} \in \mathfrak{g}^{i *}(\mathbb{K})_{x,-r_{i}}$ such that

$$
\phi_{i}\left(\mathrm{e}_{x, r_{i}: r_{i}^{+}}(Y)\right)=\Lambda\left(\left\langle X_{i}^{*}, Y\right\rangle\right)
$$

for all $Y \in \mathfrak{g}^{i}(\mathbb{K})_{x, r_{i}}$. The condition of genericity for $\phi_{i}$ implies that we may choose $X^{*}$ in the Lie algebra of a $\mathbb{K}^{\text {tame }}$-split, maximal $\mathbb{K}$-torus $T \subseteq G^{i}$ in such a way that the roots of $T$ in $G^{i+1}$ that vanish on $X_{i}^{*}$ are precisely those that appear in $G^{i}$ (see [44, §8-9]; in particular, Lemma 8.1 loc. cit.). In particular, $X_{i}^{*} \in \mathfrak{z}\left(\mathfrak{g}^{i}\right)^{*}(\mathbb{K})$. Notice that $X_{i}^{*}$ is well determined only up to translation by $\mathfrak{z}\left(\mathfrak{g}^{i}\right)^{*}(\mathbb{K})_{x,-r_{i}^{+}}$.

Definition 2.14. If $\Psi$ is a cuspidal datum such that $d=d_{\Psi}>0$, then we will write $X_{\Psi}^{*}$ for $\sum_{i=0}^{d-1} X_{i, \Psi}^{*}$, with the understanding that it is well defined only up to suitable translation. We assume that the residual characteristic of $\mathbb{K}$ is sufficiently large, so that there exists a "nice" identification of $\mathfrak{g}(\mathbb{K})$ and $\mathfrak{g}^{*}(\mathbb{K})$. (See [2, Proposition 
4.1] for details and a precise statement.) We fix any such identification, and write $\Gamma_{\Psi}$ for the element of $\mathfrak{g}(\mathbb{K})$ that corresponds to $X_{\Psi}^{*}$.

The element $\Gamma_{\Psi}$ in Definition 2.14 is the same one that occurs in 28, Definition 4.1.3(2)]. For convenience, we will sometimes write $\hat{\mu}_{\Gamma_{\Psi}}$ instead of $\hat{\mu}_{X_{\Psi}^{*}}$.

Definition 2.15. If $\Psi$ is a cuspidal datum of essential depth $r$, then we say that $\Psi$ is restricted if and only if the coset $\Gamma_{\Psi}+\mathfrak{z}\left(\mathfrak{g}^{0, \Psi}\right)(\mathbb{K})_{x_{\Psi},(-r)^{+}}$(see Definition 2.14) contains a restricted element. We will always assume that $\Gamma_{\Psi}$ itself is restricted, since we only need its coset as part of the data.

Remark 2.16. If $\Psi$ is a restricted, cuspidal datum for $G(\mathbb{K})$, then $d_{\Psi}=1$ and $G^{0, \Psi}$ is an elliptic torus in $G$. Thus we may, and do, replace any such cuspidal datum by its associated toral, very supercuspidal datum (see Remark 2.13), which we will also call a restricted datum.

Suppose that $\Psi$ and $\dot{\Psi}$ are cuspidal data such that $\pi_{\Psi} \cong \pi_{\dot{\Psi}}$. By [20, Definitions $4.19(\mathbf{F 1})$ and 5.2, and Theorem 6.7], we have, after replacing $\dot{\Psi}$ by a $G(\mathbb{K})$-conjugate (which does not affect whether or not it is restricted) and $x_{\dot{\Psi}}$ by a translate by an element of the (tensored-up) rational character lattice $X_{*}^{\mathbb{K}}\left(Z\left(G^{0, \dot{\Psi}}\right)\right) \otimes_{\mathbb{Z}} \mathbb{R}=$ $X_{*}^{\mathbb{K}}(Z(G)) \otimes_{\mathbb{Z}} \mathbb{R}$ (which does not affect the Moy-Prasad subgroups appearing below), that

$$
\begin{aligned}
d & :=d_{\Psi}=d_{\dot{\Psi}}, \\
G^{i}:=G^{i, \Psi} & =G^{i, \dot{\Psi}} \quad \forall 0 \leq i<d, \\
\vec{r} & :=\vec{r}_{\Psi}=\vec{r}_{\dot{\Psi}}, \\
x & :=x_{\Psi}=x_{\dot{\Psi}},
\end{aligned}
$$

and

$$
\prod_{i=0}^{d-1} \phi_{i, \Psi}=\prod_{i=0}^{d-1} \phi_{i, \dot{\Psi}} \quad \text { on } G^{0}(\mathbb{K})_{x, r_{d-1}} .
$$

This implies that $\Gamma_{\Psi}$ and $\Gamma_{\dot{\Psi}}$ are congruent modulo $\mathfrak{g}^{0}(\mathbb{K})_{x,-r_{d-1}^{+}}$, hence (since both are central) modulo $\mathfrak{z}\left(\mathfrak{g}^{0}\right)(\mathbb{K})_{x,-r_{d-1}^{+}}$. In particular, $\Psi$ is restricted if and only if $\dot{\Psi}$ is. Thus, the following definition makes sense.

Definition 2.17. We say that the tame, supercuspidal representation $\pi_{\Psi}$ is restricted if and only if $\Psi$ is restricted.

The characters of restricted, tame, supercuspidal representations-indeed, of all those tame, supercuspidal representations $\pi_{\Psi}$ for which $d=d_{\Psi}>0$ and $G^{d-1, \Psi} / Z(G)$ is $\mathbb{K}$-anisotropic - were computed in [6]. If, as here, one is only interested in character values near the identity, and if the residual characteristic of $\mathbb{K}$ is sufficiently large (see [28, §3.2]; in particular, Remark 3.2.1 loc. cit.), then we can also use the results of [28, which applies to a more general class of representations than the ones that we consider.

Theorem 2.18 ([28, Theorem 4.4.1] and [6, Corollary 6.7]). Let $\Psi$ be a restricted, depth-r, cuspidal datum for $G(\mathbb{K})$, and put $\pi=\pi_{\Psi}$ and $\Gamma=\Gamma_{\Psi}$ (in the notation of Definition 2.14). Then

$$
\theta_{\pi}(\mathrm{e}(Y))=\operatorname{deg}(\pi) \hat{\mu}_{\Gamma}(Y)
$$


for all regular, semisimple elements $Y$ of $\mathfrak{g}(\mathbb{K})_{r}$, where $\operatorname{deg}(\pi)$ is the formal degree of $\pi$.

We recall that formal degree was introduced by Harish-Chandra, 24, Theorem $9]$; it is an analogue of the dimension (of a finite-dimensional representation) in the Schur orthogonality relations. In characteristic zero, Haar measure may be normalized so that the formal degree of every supercuspidal representation is an integer, 24, Theorem 22.6].

\subsection{Constructible motivic, and constructible exponential, functions.}

Here we will use the theory of motivic integration and Fourier transform as developed in [10,11] (see [9, 19] for exposition). In particular, we use the following notation and terminology introduced in [10,11].

- The notation $h[m, n, r]$ stands for the functor from the category of fields containing $\mathbb{Q}$ to the category of sets that assigns to each field $L$ the set $L((t))^{m} \times L^{n} \times \mathbb{Z}^{r}$.

- All the logical formulas we consider are formulas in the language $\mathcal{L}_{\mathbb{Z}}$ (see [9, §6.7] or [19, §5]). This is the Denef-Pas language with coefficients in $\mathbb{Z} \llbracket t \rrbracket$ in the valued-field sort, combined with Presburger language for $\mathbb{Z}$. Any non-Archimedean local field $\mathbb{K}$ with a choice of a uniformizer is a structure for the language $\mathcal{L}_{\mathbb{Z}}$.

- The category Def $\mathbb{Q}_{\mathbb{Q}}$ is the category of definable subassignments of $h[m, n, r]$ for some non-negative integers $m, n, r$.

- For a definable subassignment $S \in \operatorname{Def}_{\mathbb{Q}}$, we sometimes consider the category $\operatorname{Def}_{S}$ of definable subassignments over $S$.

- For a positive integer $M$, let $\mathcal{A}_{\mathbb{Z}, M}$ stand for the collection of all finite extensions of $\mathbb{Q}_{p}$ with $p>M$, and let $\mathcal{B}_{\mathbb{Z}, M}$ stand for the collection of all fields $\mathbb{F}_{q}((t))$ with $q=p^{r}$ for some positive integer $r$ and $p>M$. We let $\mathcal{F}_{\mathbb{Z}, M}=\mathcal{A}_{\mathbb{Z}, M} \cup \mathcal{B}_{\mathbb{Z}, M}$. Define $\mathcal{F}_{\mathbb{Z}}=\bigcup_{M>0} \mathcal{F}_{\mathbb{Z}, M}$, and define $\mathcal{A}_{\mathbb{Z}}$ and $\mathcal{B}_{\mathbb{Z}}$ similarly.

- We will use the category RDef $\mathbb{Q}_{\mathbb{Q}}$; this is the category of definable subassignments of $h[0, n, 0]$ (that is, only residue-field variables are allowed in the formulas, i.e., the elements of this category are definable in the language of rings). For a subassignment $U \in \mathrm{RDef}_{\mathbb{Q}}$, given a non-Archimedean local field $\mathbb{K}$ with residue field $\mathbb{k}_{\mathbb{K}}$, we will denote the specialization of $U$ to $\mathbb{K}$ by $U\left(\mathbb{k}_{\mathbb{K}}\right)$, to emphasize that it is the set of residue-field points.

We remind the reader that a formula $\varphi$ in $\mathcal{L}_{\mathbb{Z}}$ with $m$ free variables of the valuedfield sort, $n$ free variables of the residue-field sort, and $r$ free variables of $\mathbb{Z}$-sort, and no other free variables, defines a subassignment of the functor $h[m, n, r]$; on the other hand, there exists $M>0$ (that depends on $\varphi$ ) such that given a field $\mathbb{K} \in \mathcal{F}_{\mathbb{Z}, M}$ with a choice of a uniformizer, $\varphi$ can be interpreted to give a subset of $\mathbb{K}^{m} \times \mathbb{k}_{\mathbb{K}}^{n} \times \mathbb{Z}^{r}$, where $\mathbb{k}_{\mathbb{K}}$ is the residue field of $\mathbb{K}$. For details on the specialization of formulas and subassignments, see [9, §6.7]. For a definable subassignment $S$ of $h[m, n, r]$, we denote by $S_{\mathbb{K}}$ its specialization to $\mathbb{K}$ (so that $S_{\mathbb{K}}$ is a subset of $\mathbb{K}^{m} \times \mathbb{k}_{\mathbb{K}}^{n} \times \mathbb{Z}^{r}$.

We will often call a set definable if it is obtained from a definable subassignment by specialization. We call a function definable if its graph is a definable set.

Following [11, Section 2.6], we define a point of a definable subassignment $S$ to be a pair $y=\left(y_{0}, k\left(y_{0}\right)\right)$, where $k\left(y_{0}\right)$ is a field containing $\mathbb{Q}$, and $y_{0} \in S\left(k\left(y_{0}\right)\right)$. 
If $f: Z \rightarrow S$ is a morphism (i.e., $Z$ is an object of $\operatorname{Def}_{S}$ ), one can talk about the fibre $Z_{y}$ of $Z$ at $y$, which is a subassignment of $Z$ defined using the graph of $f$ (see [11, §2.6]). Further, by considering the graph again, one can show that there exists $M_{f}>0$ such that the specialization of fibres is well defined for $\mathbb{K} \in \mathcal{F}_{\mathbb{Z}, M_{f}}$. A morphism of definable subassignments $f: Z \rightarrow S$ as above induces the map of specializations $f_{\mathbb{K}}: Z_{\mathbb{K}} \rightarrow S_{\mathbb{K}}$, and the fibres of this map are the specializations of the subassignments $Z_{y}$. We will abbreviate the notation, and denote by $Z_{y, \mathbb{K}}$ the fibre of $f_{\mathbb{K}}$ at $y \in S_{\mathbb{K}}$.

Let $S \in \operatorname{Def}_{\mathbb{Q}}$ be a definable subassignment. In [11, Cluckers and Loeser defined the ring $\mathcal{C}(S)$ of constructible motivic functions on $S$, which can be made into a $\mathbb{Q}$-algebra by tensoring with $\mathbb{Q}$ over $\mathbb{Z}$. Given a non-Archimedean local field $\mathbb{K}$ with a choice of uniformizer, we obtain, after applying specialization, the $\mathbb{Q}$-algebra of constructible $p$-adic functions $\mathcal{C}_{\mathbb{K}}$ on $S_{\mathbb{K}}$ (see [9, §6.7]). By construction, these functions are $\mathbb{Q}$-valued. For a constructible motivic function $F \in \mathcal{C}(S)$, we denote by $F_{\mathbb{K}}$ its specialization to $S_{\mathbb{K}}$. This function is well defined for fields $\mathbb{K}$ of sufficiently large residue characteristic.

Even though we cannot discuss the ring of constructible motivic functions on a subassignment $S$ in detail here, we point out that it is made up of two parts. One part is the ring of so-called Presburger functions with values in the ring

$$
A=\mathbb{Z}\left[\mathbb{L}, \mathbb{L}^{-1},\left(\left(1-\mathbb{L}^{-i}\right)^{-1}\right)_{i \in \mathbb{N}}\right] .
$$

Here $\mathbb{L}$ is a formal symbol. When we pass to the specialization of such a function $f$ to a field $\mathbb{K} \in \mathcal{F}_{\mathbb{Z}, M}$, the symbol $\mathbb{L}$ specializes to the cardinality $q$ of the residue field $\mathbb{k}_{\mathbb{K}}$. Thus, $f_{\mathbb{K}}$ is a function on $S_{\mathbb{K}}$ with values in the ring $\mathbb{Z}\left[1 / q,\left(\left(1-q^{-i}\right)^{-1}\right)_{i \in \mathbb{N}}\right]$. The other part is the Grothendieck ring of the category $\operatorname{RDef}_{S}$. When we specialize to a local field $\mathbb{K}$, an element $Z$ of $\operatorname{RDef}_{S}$ specializes to an integer-valued function that counts the numbers of $\mathbb{k}_{\mathbb{K}}$-points on fibres $Z_{s, \mathbb{K}}$. We refer to [9, Section 3.2] for details. There is also a notion of positivity for constructible motivic functions. The positive constructible motivic functions always specialize to positive-valued functions.

Remark 2.19. Note that to apply motivic integration one has to pass to Functions (introduced in [11), which are equivalence classes of functions "modulo support of smaller dimension". We will, however, deal with individual functions, thinking of them as representatives of the corresponding Functions. In our case this should not cause any confusion, since all the test functions we deal with are constant on $p$-adic balls of the same dimension as the ambient space.

Further, for a subassignment $S$ as above, the ring of motivic constructible exponential functions $\mathcal{C}^{\exp }(S)$ is defined in [10. Given a local field $\mathbb{K}$ of sufficiently large residue characteristic with uniformizer $\varpi$ and an additive character $\Lambda$ satisfying the condition

$$
\Lambda(x)=\exp \left(\frac{2 \pi i}{p} \operatorname{Tr}_{\mathbb{k}_{\mathrm{K}}}(\bar{x})\right)
$$

for $x \in \mathcal{O}_{\mathbb{K}}$, the elements of this ring specialize to what we will call ( $p$-adic) constructible exponential functions. Here, $p$ is the characteristic of $\mathbb{k}_{\mathbb{K}}, \bar{x} \in \mathbb{k}_{\mathbb{K}}$ is the reduction of $x$ modulo $\varpi$, and $\operatorname{Tr}_{\mathbb{k}_{\mathbb{K}}}$ is the trace of $\mathbb{k}_{\mathbb{K}}$ over its prime subfield (see [12, $\S 10.2]$ or [19, $\S 6.3]$ for an exposition). Note that here we make a choice of a 
square root $i$ of -1 in $\mathbb{C}$. We observe that this assumption on the character $\Lambda$ is a special case of the assumption we made in Section 2.5.

Given a field $\mathbb{K}$ as above, with uniformizer $\varpi$ and an additive character $\Lambda$ as in (44), we will consider the $\mathbb{Q}$-algebra of functions on $S_{\mathbb{K}}$ generated by the specializations of motivic constructible exponential functions. Let us denote this algebra by $\mathcal{C}_{\mathbb{K}, \Lambda}^{\exp }\left(S_{\mathbb{K}}\right)$. By definition, it contains $\mathcal{C}_{\mathbb{K}}\left(S_{\mathbb{K}}\right)$. Note that the elements of this algebra no longer have to be $\mathbb{Q}$-valued.

We will often need to talk about constructible (respectively, constructible exponential) functions on the set of $\mathbb{K}$-points of an algebraic group $H$ or its Lie algebra $\mathfrak{h}$. We observe that any affine algebraic variety $X$ (for example, $X=H$ or $X=\mathfrak{h})$ naturally gives a definable subassignment of $h[m, 0,0]$ for some $m$, and that $X_{\mathbb{K}}=X(\mathbb{K})$ (where the symbol $X$ on the left stands for the corresponding subassignment, and the one on the right for the variety), for all non-Archimedean local fields $\mathbb{K}$ of sufficiently large residue characteristic. It is in this sense that we speak below of constructible functions on $H(\mathbb{K})$ or $\mathfrak{h}(\mathbb{K})$.

Given a field $\mathbb{K}$ (with a uniformizer $\varpi$ and an additive character $\Lambda$ ), we will use the term "constructible function" (respectively, "constructible exponential function") on $S_{\mathbb{K}}$ for the elements of $\mathcal{C}_{\mathbb{K}}\left(S_{\mathbb{K}}\right)$ (respectively, of $\mathcal{C}_{\mathbb{K}, \Lambda}^{\exp }\left(S_{\mathbb{K}}\right)$ ).

2.7.1. Some definable sets. To prepare the ground for our main results, we observe that some of the maps and sets we are using are definable.

First, both our substitute exponential map e and its inverse are rational functions in the matrix entries of their arguments. Therefore, they both take definable sets to definable sets, and composition with either, takes constructible functions to constructible functions. We will rely on this fact everywhere in this paper, without further mention.

Next, we would like to show that the sets $G(\mathbb{K})_{0^{+}}^{\text {reg }}$ and $\mathfrak{g}_{0^{+}}^{\text {reg }}$ are definable. Since $\mathrm{e}\left(\mathfrak{g}(\mathbb{K})_{0^{+}}^{\mathrm{reg}}\right)=G(\mathbb{K})_{0^{+}}^{\mathrm{reg}}$, it suffices to show that $\mathfrak{g}(\mathbb{K})_{0^{+}}^{\mathrm{reg}}$ is definable. First, we observe that there is a (field-independent, as long as the residue characteristic is odd) finite set $\left\{\left(\mathfrak{g}_{z}, \overline{\mathfrak{g}}_{z}, \rho_{z}\right) \mid z \in I\right\}$ consisting of triples of:

- a definable subassignment $\mathfrak{g}_{z}$ of $\mathfrak{g}$,

- a definable subassignment $\overline{\mathfrak{g}}_{z}$ of some $h[0, n, 0]$, and

- a morphism $\rho_{z}: \mathfrak{g}_{z} \rightarrow \overline{\mathfrak{g}}_{z}$

such that, for each local, non-Archimedean field $\mathbb{K}$ and maximal parahoric subgroup $K$ of $G(\mathbb{K})$, there exist $z \in I$ and a $G(\mathbb{K})$-conjugate $K^{\prime}$ of $K$ such that the Lie algebra of $K^{\prime}$ is the specialization of $\mathfrak{g}_{z}$, the Lie algebra of the corresponding reductive quotient is the specialization of $\overline{\mathfrak{g}}_{z}$, and the specialization of $\rho_{z}$ is the reduction map. (Here, the indexing set should be thought of essentially as the set of vertices of some fixed chamber in $\mathcal{B}(G, \mathbb{K})$.) For each $z \in I$, an element $X$ of the specialization $\mathfrak{g}_{z, \mathbb{K}}$ lies in $\mathfrak{g}(\mathbb{K})_{0^{+}}$if and only if $\rho_{z}(X)^{n}=0$ for some integer $n$ that is independent of $\mathbb{K}$. Finally, since the $G(\mathbb{K})$-orbit of any definable set is definable, we conclude that $\mathfrak{g}(\mathbb{K})_{0^{+}}$, and therefore also $G(\mathbb{K})_{0^{+}}$, is definable. Since regularity is also definable (see [16, Definition 14]), we have the desired result.

From now on, we will denote by $G_{0^{+}}^{\text {reg }}$ (respectively, by $\mathfrak{g}_{0^{+}}^{\text {reg }}$ ) the definable subassignment of $G$ (respectively, of $\mathfrak{g}$ ) that specializes to $G(\mathbb{K})_{0^{+}}^{\text {reg }}$ (respectively, $\left.\mathfrak{g}(\mathbb{K})_{0^{+}}^{\mathrm{reg}}\right)$. Note that now we can talk about constructible motivic, or constructible motivic exponential functions on $G_{0^{+}}^{\mathrm{reg}}$ and $\mathfrak{g}_{0^{+}}^{\mathrm{reg}}$. 
2.8. Characters near the identity: the statements. Recall that $G$ is a symplectic or split special orthogonal group.

We need a way to handle distributions (in particular, the distribution characters) in the motivic context. The idea is to define a constructible family of constructible motivic test functions, such that their specializations form a dense subset of the space of all test functions. This is done in Section 3. Knowing the values of the distribution evaluated at the test functions from this family is equivalent to knowing the distribution itself.

We temporarily have the need to define a notion of "equivalence of representations", that, a priori, is even less refined than $r$-equivalence. We will prove in Lemma 3.4 that this is in fact the same notion as our notion of $r$-equivalence.

Definition 2.20. Let $\mathbb{K} \in \mathcal{F}_{\mathbb{Z}}$. We call two representations $\pi$ and $\pi^{\prime}$ of $G(\mathbb{K})$ definably $r$-equivalent (or say that they are in the same definable $r$-equivalence class) if there exists a constructible family of constructible motivic functions $f_{a}$, indexed by a parameter $a$ from some definable subassignment $S$, such that the following conditions are satisfied:

(1) The support of the specialization $f_{a, \mathbb{K}}$ of $f_{a}$ to $\mathbb{K}$ is contained in $G(\mathbb{K})_{r}^{\text {reg, }}$, for every $a \in S_{\mathbb{K}}$.

(2) There exists a family of definable subassignments $\mathcal{K}_{n}$, such that:

- $\bigcup_{n>0} \mathcal{K}_{n}=G_{0^{+}}^{\text {reg }}$, and

- for every $n>0$, the $\mathbb{C}$-span of the functions from the family $f_{a, \mathbb{K}}$ with supports contained in $\mathcal{K}_{n, \mathbb{K}}$ is dense in $C_{c}^{\infty}\left(G(\mathbb{K})_{r}^{\mathrm{reg}} \cap \mathcal{K}_{n, \mathbb{K}}\right)$ (with respect to the sup-norm).

(3) The distribution characters of $\pi$ and $\pi^{\prime}$ coincide on the functions $f_{a, \mathbb{K}}$, i.e., we have

$$
\Theta_{\pi}\left(f_{a, \mathbb{K}}\right)=\Theta_{\pi^{\prime}}\left(f_{a, \mathbb{K}}\right)
$$

for all $a \in S_{\mathbb{K}}$.

Theorem 2.21. There exists an integer $M>0$ such that the following three statements hold for every non-Archimedean local field $\mathbb{K} \in \mathcal{F}_{\mathbb{Z}, M}$ and $r>0$.

(1) There exists a definable, in the language of rings, subassignment $B_{\mathfrak{g},-r} \in$ $R D e f_{\mathbb{Q}}$ that parameterizes the definable r-equivalence classes of restricted representations of $G(\mathbb{K})$ of minimal depth $r$, in the sense that there is a one-to-one (well-defined) map from the set of definable r-equivalence classes of such representations onto $B_{\mathfrak{g},-r}\left(\mathbb{k}_{\mathbb{K}}\right)$.

(2) There exists a positive constructible motivic function $Q_{\mathfrak{g},-r}$ on $B_{\mathfrak{g},-r}$, and for every constructible motivic test function $f$ such that the support of its specialization $f_{\mathbb{K}}$ is contained in $G(\mathbb{K})_{r}^{\mathrm{reg}}$, there exists a constructible motivic exponential function $H^{f}$ on the definable subassignment $B_{\mathfrak{g},-r}$, and a positive integer $M_{f}$ (which might depend on $f$ ), such that for every field $\mathbb{K}$ with residue characteristic bigger than $M+M_{f}$ we have

$$
\frac{1}{\operatorname{deg}(\pi)} \Theta_{\pi}\left(f_{\mathbb{K}}\right)=\frac{H_{\mathbb{K}}^{f}(x)}{Q_{\mathfrak{g},-r, \mathbb{K}}(x)},
$$

for every restricted, supercuspidal, minimal-depth-r representation $\pi$ with corresponding point $x \in B_{\mathfrak{g},-r}\left(\mathbb{K}_{\mathbb{K}}\right)$. 
(3) Let $\left\{f_{a}\right\}_{a \in S}$ be a family of constructible motivic functions on $G(\mathbb{K})$ satisfying Definition 2.20 (11). Then $\Theta_{\pi}\left(f_{a, \mathbb{K}}\right)$ is a specialization of a constructible motivic exponential function of $a$.

Remark 2.22. Note that we consider only representations of minimal depth above. Since every representation has a twist by a linear character of $G(\mathbb{K})$ that is of minimal depth, this is not a serious restriction. Since the necessary twist is trivial on a sufficiently small neighbourhood of the identity, we could remove the restriction by considering germs of characters (rather than their restrictions to a specified neighbourhood of the identity). Another approach would be to incorporate the character of $G$ into the language, so that we could use it directly in our description of character values. We believe that our present approach is the simplest.

Let $G$ be a symplectic or special orthogonal group, as above. According to the above theorem, there exists $M>0$ such that for every $\mathbb{K} \in \mathcal{F}_{\mathbb{Z}, M}$, the definable $r$-equivalence classes (in the sense of Definition 2.20) of restricted, depth- $r$, supercuspidal representations of $G(\mathbb{K})$ may be parameterized by points $x \in B_{\mathfrak{g},-r}\left(\mathbb{k}_{\mathbb{K}}\right)$. Hence, if $\pi$ is a restricted, supercuspidal representation of $G(\mathbb{K})$ of minimal depth $r$ whose parameter in $B_{\mathfrak{g},-r}\left(\mathbb{k}_{\mathbb{K}}\right)$ is $x$, then, as we will prove later in Lemma 3.4. we may write unambiguously $\Theta_{x}$ for the restriction to $G(\mathbb{K})_{r}^{\text {reg }}$ of the distribution character of $\pi$. We also denote the restriction of $\theta_{\pi}$ to $G(\mathbb{K})_{r}^{\text {reg }}$ by $\theta_{x}$. Finally, we observe that the germ of the character determines the formal degree of the representation; hence, all representations of $G(\mathbb{K})$ that lie in the same definable equivalence class corresponding to a point $x \in B_{\mathfrak{g},-r}\left(\mathbb{K}_{\mathbb{K}}\right)$ have the same formal degree, which we will denote by $\operatorname{deg}(x)$.

Theorem 2.23. Let $G$ and $r>0$ be as above. Let $M$ be the constant from Theorem 2.21, and let $Q_{\mathfrak{g},-r}$ be the constructible motivic function on $B_{\mathfrak{g},-r}$ from the same theorem. Then there exists a constructible motivic exponential function $F$ on $B_{\mathfrak{g},-r} \times G_{0^{+}}^{\mathrm{reg}}$ such that, for every local field $\mathbb{K} \in \mathcal{F}_{\mathbb{Z}, M}$ and every $x \in B_{\mathfrak{g},-r}\left(\mathbb{K}_{\mathbb{K}}\right)$,

$$
\frac{1}{\operatorname{deg}(x)} \theta_{x}(g)=\frac{F_{\mathbb{K}}(x, g)}{Q_{\mathfrak{g},-r, \mathbb{K}}(x)}
$$

for all $g \in G(\mathbb{K})_{r}^{\mathrm{reg}}$ (where $F_{\mathbb{K}}$ is the specialization of $F$ ). In particular, each function $\theta_{x}$ on $G(\mathbb{K})_{r}^{\mathrm{reg}}$ coincides with the restriction to $G(\mathbb{K})_{r}^{\mathrm{reg}}$ of a specialization of a constructible motivic exponential function.

Remark 2.24. Note that both the formal degree of a representation, and the Fourier transform of an elliptic orbital integral (here we mean not the distribution, but the function that represents it), depend on the choice of Haar measure on $G(\mathbb{K})$ (really, on $G(\mathbb{K}) / Z(G)(\mathbb{K})$ and $G(\mathbb{K}) / T(\mathbb{K})$, for $T$ an elliptic $\mathbb{K}$-torus, respectively; but remember that we are in the semisimple case). On the other hand, the product of the two is well defined (i.e., independent of Haar measure), so that the equality asserted in Theorem 2.18 makes sense. (The Fourier transform also depends on a choice of Haar measure on $\mathfrak{g}(\mathbb{K})$, but there is a natural choice in this setting, namely, the one for which, with the obvious notation, $\hat{\hat{f}}(X)=f(-X)$ for all $X \in \mathfrak{g}(\mathbb{K})$ and $f \in C_{c}^{\infty}(\mathfrak{g}(\mathbb{K}))$.) Therefore, we may, and do, fix the Haar measure that is compatible with motivic integration, that is, the measure that coincides with Serre-Oesterlé measure on $G\left(\mathcal{O}_{\mathbb{K}}\right)$.

Theorem 2.23 shows that, for each $x$, the function $\theta_{x}$ is a specialization of a constructible motivic exponential function; but the dependence on the formal degree 
at present prevents us from showing that the function $\theta_{x}$ depends definably on $x$ (though we believe that this is the case). Here, we only show that $\frac{1}{\operatorname{deg}(x)} \theta_{x}$ depends definably on $x$.

These theorems will be proved in Sections 4.3. 4.4 and 4.5, respectively.

2.9. Interpretation in terms of $L$-packets. As we will see, the first statement in Theorem 2.21 in fact gives a parameterization of restricted, depth- $r$ representations up to $r$-equivalence, in the sense of Definition 2.9. The strategy for proving it is to express the restriction of the character of such a representation as a Fourier transform of an orbital integral (see Theorem 2.18), and then to construct the parameter space $B_{\mathfrak{g},-r}$ for the orbital integrals that arise this way using the ideas from [14. The parameter space $B_{\mathfrak{g},-r}$ comes with a projection to $S_{\mathfrak{g},-r}$ which is a variety whose residue-field points parameterize the stable thickened orbits of restricted elements in $\mathfrak{g}(-r, \mathbb{K})$; see Theorem 4.2 .

Let $\Psi$ be the cuspidal datum that gives rise to $\pi$, and $\Gamma_{\Psi}$ the element defined in Definition 2.14, Then

$$
\Theta_{\pi}(f)=\operatorname{deg}(\pi) \hat{\mu}_{\Gamma_{\Psi}}\left(f \circ \mathrm{e}^{-1}\right)=\operatorname{deg}(\pi) \mu_{\Gamma_{\Psi}}\left(\widehat{f \circ \mathrm{e}^{-1}}\right),
$$

for all $f \in C_{c}^{\infty}\left(G(\mathbb{K})^{\text {reg }}\right)$. As we will see from the construction of the subassignment $B_{\mathfrak{g},-r}$ in Section 4.2, the elements $\Gamma_{\Psi}$ are grouped according to stable conjugacy at first, before being grouped according to conjugacy. Namely, we first find a point in $S_{\mathfrak{g},-r}$ that corresponds to the stable thickened orbit $\mathcal{O}_{\Psi}$ of $\Gamma_{\Psi}$; the fibre of $B_{\mathfrak{g},-r}$ over that point parameterizes the thickened orbits in $\mathcal{O}_{\Psi}$.

Therefore, it is natural to ask whether one can detect stable conjugacy of the elements $\Gamma_{\Psi}$ in terms of the cuspidal data that gave rise to them. This question is addressed in this section.

In [36], Reeder constructs some candidates for $L$-packets of positive-depth, supercuspidal representations on $G(\mathbb{K})$ in case $G$ is unramified.

Following [15, §9.4], we introduce the notion of stable conjugacy in order to describe the packet. The following definition would not change if we allowed $g$ to range over the $\mathbb{K}^{\text {sep }}$-points of $G$, for a fixed separable closure $\mathbb{K}^{\mathrm{sep}} / \mathbb{K}$, but it is more convenient for us to phrase it as we have done.

Definition 2.25. We say that two toral, very supercuspidal data $(T, \phi)$ and $(\dot{T}, \dot{\phi})$ for $G(\mathbb{K})$ (respectively, two regular, semisimple elements $X, X^{\prime} \in \mathfrak{g}(\mathbb{K})$ ) are stably conjugate if and only if there exists some element $g \in G\left(\mathbb{K}^{\text {unr }}\right)$ such that $\operatorname{Int}(g) T(\mathbb{K})=\dot{T}(\mathbb{K})$ and $\dot{\phi} \circ \operatorname{Int}(g)=\phi\left(\right.$ respectively, $\left.\operatorname{Ad}(g) X=X^{\prime}\right)$. Then we put

$$
\Pi(T, \phi):=\{\pi(\dot{T}, \dot{\phi}) \mid(\dot{T}, \dot{\phi}) \text { is stably conjugate to }(T, \phi)\} \text {. }
$$

The sets $\Pi(T, \phi)$ are the conjectural positive-depth, supercuspidal $L$-packets of Reeder (cf. [36, §6.6]).

Remark 2.26. Note that, if $g \in G\left(\mathbb{K}^{\text {unr }}\right)$ is as in Definition 2.25 and $r \in \widetilde{\mathbb{R}}_{\geq 0}$, then

$$
\operatorname{Int}(g)\left(T(\mathbb{K})_{r}\right) \subseteq \operatorname{Int}(g)\left(T\left(\mathbb{K}^{\text {unr }}\right)_{r}\right) \cap \dot{T}(\mathbb{K})=\dot{T}\left(\mathbb{K}^{\text {unr }}\right)_{r} \cap \dot{T}(\mathbb{K})=\dot{T}(\mathbb{K})_{r}
$$

(by the definition of the filtrations). The same argument, with the rôles of $T$ and $\dot{T}$ reversed, shows that we have equality; i.e., that $\operatorname{Int}(g)\left(T(\mathbb{K})_{r}\right)=\dot{T}(\mathbb{K})_{r}$. In particular, the depths of $\phi$ and $\dot{\phi}$ are the same. 
It is reasonable to believe that a similar parameterization to Reeder's will produce $L$-packets for toral, very supercuspidal representations even if the relevant tori do not split over an unramified extension (even, given a suitable definition of stable conjugacy, if the cuspidal datum is not toral!).

Proposition 2.27. Suppose that $\Psi=(T, \phi)$ and $\dot{\Psi}=(\dot{T}, \dot{\phi})$ are stably conjugate (see Definition 2.25), restricted (hence toral, very supercuspidal) data of essential depth $r$. Then, in the notation of Definition 2.14, $\mathcal{O}_{-r}^{s t}\left(\Gamma_{\Psi}\right)=\mathcal{O}_{-r}^{s t}\left(\Gamma_{\dot{\Psi}}\right)$.

Proof. Suppose that the conjugacy of $\Psi$ and $\dot{\Psi}$ is afforded by the element $g \in$ $G\left(\mathbb{K}^{\text {unr }}\right)$. It suffices to show that, in the notation of Definition 2.14 $\operatorname{Ad}^{*}(g) X_{\Psi}^{*} \equiv$ $X_{\dot{\Psi}}^{*}\left(\bmod \dot{\mathfrak{t}}^{*}(\mathbb{K})_{(-r)^{+}}\right)$. (Recall that $X_{\Psi}^{*}$ is the element of $\mathfrak{g}^{*}(\mathbb{K})$ to which the element $\Gamma_{\Psi} \in \mathfrak{g}(\mathbb{K})$ corresponds.)

Put $\dot{X}_{\Psi}^{*}=\operatorname{Ad}^{*}(g) X_{\Psi}^{*}$. The images in $\mathcal{B}(G, \mathbb{K})$ of $\mathcal{B}(T, \mathbb{K})$ and $\mathcal{B}(\dot{T}, \mathbb{K})$ are singletons. Write $x$ and $\dot{x}$, respectively, for their elements. Since $X_{\Psi}^{*} \in \mathfrak{t}^{*}(\mathbb{K})$, we have that $\dot{X}_{\Psi}^{*} \in \dot{\mathfrak{t}}^{*}(\mathbb{K})$. Fix $\dot{Y} \in \dot{\mathfrak{t}}(\mathbb{K})_{r}$. Reasoning as in Remark 2.26 shows that $Y:=\operatorname{Ad}(g)^{-1} \dot{Y}$ lies in $\mathfrak{t}(\mathbb{K})_{r}$. Since $\mathrm{e}_{\dot{x}, r: r^{+}}(\dot{Y})=\operatorname{Int}(g) \mathrm{e}_{x, r: r^{+}}(Y)$ by our choice of e (which, remember, admits an equivariance property with respect to conjugation, not just by $G(\mathbb{K})$, but also by $G\left(\mathbb{K}^{\text {unr }}\right)$; see page 535$)$, we have by the definition of the elements $X_{\Psi}^{*}$ and $\dot{X}_{\Psi}^{*}$ that

$$
\begin{aligned}
\Lambda\left(\left\langle X_{\Psi}^{*}, \dot{Y}\right\rangle\right)=\dot{\phi}\left(\mathrm{e}_{\dot{x}, r: r^{+}}(\dot{Y})\right)=\dot{\phi} & \left(\operatorname{Int}(g) \mathrm{e}_{x, r: r^{+}}(Y)\right) \\
& =\phi\left(\mathrm{e}_{x, r: r^{+}}(Y)\right)=\Lambda\left(\left\langle X_{\Psi}^{*}, Y\right\rangle\right)=\Lambda\left(\left\langle\dot{X}_{\Psi}^{*}, \dot{Y}\right\rangle\right) .
\end{aligned}
$$

Since $\dot{Y} \in \dot{\mathfrak{t}}(\mathbb{K})_{r}$ was arbitrary, we obtain the desired congruence.

This proposition shows that as we assign parameters in $B_{\mathfrak{g},-r}\left(\mathbb{K}_{\mathbb{K}}\right)$ to the representations, Reeder's conjectural $L$-packets (cf. Definition 2.25) are preserved, in the sense that the parameters for representations from the same $L$-packet project to the same point in $S_{\mathfrak{g},-r}\left(\mathbb{K}_{\mathbb{K}}\right)$.

\section{Distributions AND BAD PRIMEs}

We would like to consider distributions in the context of motivic integration. In this paper, we express the value of the distribution character $\Theta_{\pi}$ at a test function $f \in C_{c}^{\infty}(G(\mathbb{K}))$ as a motivic integral, when the support of $f$ is contained in a sufficiently small neighbourhood of the identity element. Typically, as we perform motivic integration, we need to discard a finite number of primes. Therefore, there arises the problem that if for different test functions we need to discard different primes, then we might not be able to make any conclusion about the distribution on the whole for any fields. In this section we show that this is in fact not the case.

Note that Theorem 2.23, concerning Harish-Chandra characters, decreases the need to discuss distribution characters. We include this section nonetheless, partly because it allows us to establish Theorem 2.21(1), but also because we hope that the simple observations explored here will be helpful for many similar situations in other contexts.

3.1. A dense family of definable functions. Let $S_{d}$ be the definable subassignment of $h[d, 0,0]$ defined by $K \mapsto K \llbracket t \rrbracket^{d}$. Here, as everywhere in this paper, we use the language with coefficients in $\mathbb{Z} \llbracket t \rrbracket$ in the valued-field sort. 
Let us consider the $\mathbb{C}$-algebra generated by the specializations of constructible functions, that is,

$$
\mathcal{C}_{\mathbb{K}, \mathbb{C}}\left(\mathbb{A}^{d}(\mathbb{K})\right):=\mathcal{C}_{\mathbb{K}}\left(\mathbb{A}^{d}(\mathbb{K})\right) \otimes_{\mathbb{Z}} \mathbb{C} .
$$

Similarly, for any definable subassignment $S$, we can consider

$$
\mathcal{C}_{\mathbb{K}, \mathbb{C}}\left(S_{\mathbb{K}}\right):=\mathcal{C}_{\mathbb{K}}\left(S_{\mathbb{K}}\right) \otimes_{\mathbb{Z}} \mathbb{C}
$$

(see [12, §2.9] for a discussion of why tensoring with $\mathbb{C}$ is compatible with motivic integration and the definition of the class of integrable functions).

The proposition below holds for a general base field, but we state it for $k=\mathbb{Q}$ for simplicity, since this is all we need.

Proposition 3.1. Fix a positive integer $d$. Let $\eta_{a} \in \mathcal{C}\left(S_{d}\right) \otimes \mathbb{C}$ be the family of constructible motivic functions, where $a=\left(a_{1}, \ldots, a_{d}, r_{1}, \ldots, r_{d}\right)$ with $r_{i} \geq 0$ is an element of $\mathbb{Z} \llbracket t \rrbracket^{d} \times \mathbb{Z}^{d}$, defined by

$$
\eta_{a}\left(x_{1}, \ldots, x_{d}\right)= \begin{cases}1, & \operatorname{ord}\left(x_{i}-a_{i}\right) \geq r_{i} \quad \text { for } i=1, \ldots, d, \\ 0, & \text { otherwise. }\end{cases}
$$

Suppose $\left\{f_{\alpha}\right\}_{\alpha \in A}$ is a definable family of definable functions on $S_{d}$, parameterized by some definable subassignment $A$. Then there exists $M>0$ such that for all fields $\mathbb{K} \in \mathcal{F}_{\mathbb{Z}, M}$ there is a well-defined specialization $\eta_{a, \mathbb{K}}$ that is a function on $\mathcal{O}_{\mathbb{K}}^{d}$, and the motivic integral $\int_{S_{d}} f_{\alpha} \eta_{a}$ specializes to $\int_{S_{d, \mathbb{K}}} f_{\alpha} \eta_{a, \mathbb{K}}$.

Proof. This proposition follows directly from the specialization theorems (with parameters) 9, Theorems 6.9 and 7.3], since we can simply consider the $a_{i}$ and $r_{i}$ as extra variables of the functions $\eta_{a}$.

Proposition 3.2. Let $S$ be a definable subassignment of $h[d, 0,0]$ for some $d>0$. Assume that $S$ is contained in the subassignment defined by $\operatorname{ord}\left(x_{i}\right) \geq N, i=$ $1, \ldots, d$, for some $N \in \mathbb{Z}$. Let $\mathrm{CF}\left(S_{\mathbb{K}}\right)$ be the algebra of complex-valued continuous functions on $S_{\mathbb{K}}$ (with sup-norm). Then there exists $M$ such that for any $\mathbb{K} \in \mathcal{F}_{\mathbb{Z}, M}$, the algebra $\mathcal{C}_{\mathbb{K}, \mathbb{C}}\left(S_{\mathbb{K}}\right) \cap \mathrm{CF}\left(S_{\mathbb{K}}\right)$ is dense in $\mathrm{CF}\left(S_{\mathbb{K}}\right)$. In particular, if two distributions defined on $S_{\mathbb{K}}$ and continuous with respect to the sup-norm coincide on $\mathcal{C}_{\mathbb{K}, \mathbb{C}}\left(S_{\mathbb{K}}\right)$, then they are identical.

Proof. By multiplying $x_{i}$ by $t^{-N}$ in the case $N<0$, one can make a definable embedding $\phi: S \rightarrow S_{d}$, where $S_{d}$ is the definable subassignment $K \mapsto K \llbracket t \rrbracket^{d}$ as above. Let $\left\{\eta_{a}\right\}_{a \in \mathbb{Z} \llbracket t \rrbracket^{d} \times \mathbb{Z}^{d}}$ be the family from Proposition 3.1. It is clear that the family $\left\{\xi_{a}=\phi^{*}\left(\eta_{a}\right)\right\}$ separates points in $S_{\mathbb{K}}$. The statement now follows from the Stone-Weierstrass theorem.

Though we do not currently have a general framework for "constructible motivic distributions", Proposition 3.2 allows us to handle many distributions in the context of motivic integration.

Remark 3.3. The theory of motivic integration recently developed by E. Hrushovski and D. Kazhdan 25,41,43 includes distributions, and their treatment of distributions by evaluating them on definable families of test functions is similar to ours; however, since the specialization of that theory to $p$-adic integration has not yet been completely worked out, we have to include the present discussion. 
3.2. Distribution characters. Now we are ready to prove that definable $r$-equivalence of representations (see Definition 2.20) coincides with $r$-equivalence (see Definition 2.9).

Lemma 3.4. Let $\mathbb{K} \in \mathcal{F}_{\mathbb{Z}}$ be a field. Then two r-restricted supercuspidal representations $\pi$ and $\pi^{\prime}$ of $G(\mathbb{K})$ are r-equivalent if and only if they are definably $r$-equivalent.

Proof. Obviously, $r$-equivalence implies definable $r$-equivalence. Let us prove the converse. Let $\pi$ and $\pi^{\prime}$ be definably $r$-equivalent representations. We need to show that the restrictions of the functions $\theta_{\pi}$ and $\theta_{\pi^{\prime}}$ coincide on $G(\mathbb{K})_{r}^{\text {reg }}$. Suppose this is not so, so that there exists $x_{0} \in G(\mathbb{K})_{r}^{\text {reg }}$ such that $\theta_{\pi}\left(x_{0}\right) \neq \theta_{\pi^{\prime}}\left(x_{0}\right)$. Since both $\theta_{\pi}$, and $\theta_{\pi^{\prime}}$ are locally constant on $G(\mathbb{K})_{r}^{r \text { reg }}$, there exists a neighbourhood $U$ of $x_{0}$ such that these functions are constant on $U$ with different values. Let $\mathbf{1}_{U}$ be the characteristic function of $U$.

By the definition of definable equivalence, there exists a definable compact set $\mathcal{K}_{n, \mathbb{K}}$ that contains $U$, and such that the span of the set of functions from the family $f_{a, \mathbb{K}}$ of Definition 2.20 with supports contained in $\mathcal{K}_{n, \mathbb{K}}$ is dense in $C_{c}^{\infty}\left(G(\mathbb{K})_{r}^{\text {reg }} \cap \mathcal{K}_{n, \mathbb{K}}\right)$. Thus, on the one hand, for every $\varepsilon$, there exists a function $f_{\varepsilon}$ in the span of this family, with $\operatorname{supp}\left(f_{\varepsilon}\right) \subset \mathcal{K}_{n, \mathbb{K}}$, such that $\Theta_{\pi}\left(f_{\varepsilon}\right)=\Theta_{\pi^{\prime}}\left(f_{\varepsilon}\right)$, and such that

$$
\sup _{\mathcal{K}_{n, \mathbb{K}}}\left|f_{\varepsilon}-\mathbf{1}_{U}\right|<\varepsilon
$$

On the other hand, as $\varepsilon$ gets small, then $\Theta_{\pi}\left(f_{\varepsilon}\right)$ has to get arbitrarily close to $\theta_{\pi}\left(x_{0}\right) \operatorname{vol}(U)$, whereas $\Theta_{\pi^{\prime}}\left(f_{\varepsilon}\right)$ has to get close to $\theta_{\pi^{\prime}}\left(x_{0}\right) \operatorname{vol}(U)$, leading to a contradiction.

3.2.1. A definable family of test functions. Finally, in order to talk about character distributions in the motivic context, we construct a definable family of definable functions, essentially, made from the family of functions $\left\{\eta_{a}\right\}_{a \in \mathbb{Z} \llbracket t \rrbracket^{d} \times \mathbb{Z}^{d}}$ of Proposition 3.1, to test the distributions for definable equivalence. Here we have to be somewhat careful about the number of restrictions we impose on the residue characteristic. As shown in Section 2.7.1 there is a definable subassignment $G_{0^{+}}^{\text {reg }}$ of $G$ such that $G_{0^{+}, \mathbb{K}}^{\mathrm{reg}}=G(\mathbb{K})_{0^{+}}^{\mathrm{reg}}$ for all $\mathbb{K} \in \mathcal{F}_{\mathbb{Z}, M}$, with some $M>0$ that depends only on $G$. The number of conjugacy classes of maximal compact subgroups in $G(\mathbb{K})$ is finite, and bounded by a constant that depends only on $G$ and is independent of $\mathbb{K}$. One can choose a definable maximal compact subgroup in each conjugacy class (given $G$, it is possible to write down explicit conditions on the matrix entries defining these compact subgroups). Let $\Omega$ be a definable subassignment of $G$ such that $\Omega_{\mathbb{K}}$ is a union of definable maximal compact subgroups, and every compact element in $G(\mathbb{K})$ is conjugate to an element of $\Omega_{\mathbb{K}}$. Let $\Omega_{n}$ be the subassignment of $\Omega$ defined by ord $\left(D^{\mathfrak{g}}\left(\mathrm{e}^{-1}(g)\right)\right) \leq n$, where $D^{\mathfrak{g}}$ is Weyl discriminant. Then $\left\{\Omega_{n}\right\}$ is a definable family of definable compact subsets of $\Omega \cap G^{\text {reg }}$. Finally, let $\left\{\xi_{a, n}\right\}$ be the definable family of definable functions from Proposition 3.2 for the subassignment $\Omega_{n}$. Let $M_{\xi}$ be a constant such that for all $n>0$, for all $\mathbb{K} \in \mathcal{F}_{\mathbb{Z}, M_{\xi}}$, the family $\left\{\xi_{a, n}\right\}$ specializes to a dense family of functions with supports contained in $\Omega_{n, \mathbb{K}}$.

We will use the family $\left\{\xi_{a, n}\right\}$ in Section 4.3 in order to establish definable equivalence of characters. We will also need 
Lemma 3.5. Suppose $\left\{f_{\alpha}\right\}_{\alpha \in A}$ is a constructible family of constructible motivic functions. Then there exists $M \geq M_{\xi}$ (that depends on the family $\left\{f_{\alpha}\right\}$ ), such that $\int_{G} f_{\alpha} \xi_{a, n}$ specializes to $\int_{G(\mathbb{K})} f_{\alpha, \mathbb{K}} \xi_{a, n, \mathbb{K}}$ for all $\mathbb{K} \in \mathcal{F}_{\mathbb{Z}, M}$.

Proof. This follows from the specialization theorems [9, Theorems 6.9 and 7.3].

\section{ThE PARAMETERIZATION OF RESTRICTED ORBITAL INTEGRALS}

4.1. Waldspurger's parameterization of semi-simple orbits, and $r$-reductions. We only sketch this parameterization, referring to [40, §I.7] for details. We will use the language of stable conjugacy and stable orbits throughout; see Definition 2.25

The orbits of regular semisimple elements in $\mathfrak{g}(\mathbb{K})$ are parameterized using quadruples $\left(I,\left(F_{i} / F_{i}^{\#}\right),\left(a_{i}\right),\left(c_{i}\right)\right)$, with:

- A finite set $I$.

- For each $i \in I$, a finite extension $F_{i}^{\#}$ of $\mathbb{K}$, and a degree-2 commutative algebra $F_{i}$ over $F_{i}^{\#}$. In all cases, $F_{i}$ is either a quadratic extension of $F_{i}^{\#}$ or is isomorphic to a direct sum of two copies of $F_{i}^{\#}$. The set of indices $i$ such that $F_{i}$ is a field is denoted by $I^{*}$. This set is crucial for indexing orbits inside a stable orbit.

- For each $i \in I$, an element $a_{i} \in F_{i}^{\times}$satisfying the condition $\tau_{i}\left(a_{i}\right)=-a_{i}$, where $\tau_{i}$ is the non-trivial involution on $F_{i}$ over $F_{i}^{\#}$, and such that $a_{i}$ generates $F_{i}$ over $\mathbb{K}$.

- For each $i \in I$, an element $c_{i} \in F_{i}^{\times}$such that $\tau_{i}\left(c_{i}\right)=-c_{i}$.

There are further conditions each datum must satisfy (see [40, §I.7, (2), (4)]). Loosely speaking, the extensions $F_{i}$ and the elements $a_{i}$ determine the stable conjugacy class of $X$, and the elements $c_{i}$ are responsible for individual $\mathbb{K}$-orbits inside a stable orbit.

To emphasize the rôle of the algebras $F_{i}$ and the set $I^{*}$, we change the notation slightly, and label the data by $\left(I, I^{*},\left(F_{i} / F_{i}^{\#}\right),\left(a_{i}\right),\left(c_{i}\right)\right)$. Following [40, we assume that $c_{i}$ is only given for $i \in I^{*}$.

We recall some of the details of the correspondence (see the proof of [14, Theorem 4.6]). Since we will use these facts in the proof of Proposition 4.6(3) below in an essential way, we single them out as a proposition.

Proposition 4.1 ([14, Theorem 4.6, Corollary 4.5]). Let $\mathfrak{g}$ be a symplectic or split special orthogonal group and let $\mathbb{K}$ be a fixed local field. Suppose that $X, X^{\prime} \in$ $\mathfrak{g}(r, \mathbb{K})$, and let

$$
\left(I, I^{*}, F_{i},\left(a_{i}\right),\left(c_{i}\right)\right) \quad \text { and } \quad\left(I^{\prime}, I^{\prime *}, F_{i}^{\prime},\left(a_{i}^{\prime}\right),\left(c_{i}^{\prime}\right)\right)
$$

be the corresponding data. Then:

(1) The element $X^{\prime}$ is stably conjugate to some element $Y$ that lies in the centralizer of an element $X$, if and only if there exists a bijection $\psi: I \rightarrow I^{\prime}$ such that $F_{i}$ is isomorphic to $F_{\psi(i)}^{\prime}$, and the isomorphism intertwines $\tau_{i}$ and $\tau_{\psi(i)}^{\prime}$, for all $i \in I$.

(2) If $[X]_{r}=\left[X^{\prime}\right]_{r}$, then the centralizers of $X$ and $X^{\prime}$ are stably conjugate; there exists a bijection $\psi: I \rightarrow I^{\prime}$ such that $F_{i} \cong F_{\psi(i)}^{\prime}$ as above, and, if we identify $F_{\psi(i)}^{\prime}$ with $F_{i}$, then the elements $a_{i}$, and $a_{i}^{\prime}$ have the same valuation and angular component for all $i \in I$. 
(3) If $X$ and $X^{\prime}$ are stably conjugate, then, for all $i \in I$, the isomorphism $F_{i} \rightarrow F_{\psi(i)}^{\prime}$ above may be chosen to carry $a_{i}$ to $a_{i}^{\prime}$.

4.2. The parameterizing subassignment for orbits of restricted elements. Our construction relies heavily on the results of [14. In particular, we use the following theorem.

Theorem 4.2 ([14, Theorem 2.6]). There exists $M>0$ and a variety $S_{\mathfrak{g}, r}$ over $\mathbb{Z}[1 / M]$ such that for all $\mathbb{K} \in \mathcal{F}_{\mathbb{Z}, M}$, there is a natural bijection between the $r$ equivalence classes in $\mathfrak{g}(r, \mathbb{K})$, and the points of $S_{\mathfrak{g}, r}\left(\mathbb{K}_{\mathbb{K}}\right)$.

In the language used in this paper, we will think of $S_{\mathfrak{g}, r}$ as a subassignment that is definable in the language of rings, and thus is an element of RDef $\mathbb{Q}$.

Proposition 4.1 implies that if $X \in \mathfrak{g}(r, \mathbb{K})$, the cardinality of the set $I^{*}(X)$ depends only on the image of $X$ in $S_{\mathfrak{g}, r}\left(\mathbb{k}_{\mathbb{K}}\right)$. Therefore, for $x \in S_{\mathfrak{g}, r}\left(\mathbb{k}_{\mathbb{K}}\right)$, we can talk about the cardinality $\left|I^{*}(x)\right|$.

Lemma 4.3. Let $k$ be a positive integer. The set of points $x$ of $S_{\mathfrak{g}, r}$ such that $\left|I^{*}(x)\right|=k$ is definable. We denote the corresponding definable subassignment by $A_{k, \mathfrak{g}, r}$.

Proof. Note that the cardinality of $I^{*}(X)$ equals the number of irreducible factors in the characteristic polynomial of $X$ that are even polynomials (cf. [14, §3.3] and [16. §3.2]). It is easy to see that there is a (rather cumbersome) formula in the language of rings defining the set of elements $X$ such that exactly $k$ irreducible factors of the characteristic polynomial of $X$ are even.

We can use the coefficients of the characteristic polynomial of $X$ as free variables in this formula (since they are expressions in the matrix entries of $X$ ). Let $P_{X}$ be the characteristic polynomial of $X$. In the spirit of [16], we think of $P_{X}$ as a collection of terms that are expressions in the entries of $X$. Then our condition is given by the disjunction of formulas $\varphi_{l}$, as $l$ ranges from 0 to $n_{G}$ :

$$
\begin{aligned}
\varphi_{l}\left(P_{X}\right) & ={ }^{\prime} \exists f_{1}, \ldots, f_{k}, g_{1}, \ldots, g_{l}: P_{X}=f_{1} \ldots f_{k} g_{1} \ldots g_{l} \\
& \wedge\left(f_{1}, \ldots, f_{k} \text { are even and non-constant }\right) \\
& \wedge\left(g_{1}, \ldots, g_{l} \text { are not even }\right)^{\prime}, \quad \text { for } l>0 ; \\
\varphi_{0}\left(P_{X}\right) & =' \exists f_{1}, \ldots, f_{k}: P_{X}=f_{1} \ldots f_{k} \wedge\left(f_{1}, \ldots, f_{k} \text { are even and nonconstant }\right) '
\end{aligned}
$$

Clearly, the condition that a polynomial is even can be expressed by a ring formula in its coefficients. Note that within the language of rings, one handles degree- $m$ polynomials as $(m+1)$-tuples of coefficients, and therefore each formula quantified over polynomials $f$, in fact should be interpreted as a disjunction of formulas, one for each possible degree of $f$ (up to degree $n_{G}$, in the notation of \$1), each with the corresponding number of variables.

Now we are ready to construct the subassignment that will parameterize the thickened orbits of restricted, depth- $r$ elements.

Definition 4.4. For each positive integer $k$, let $T_{k}$ be the subassignment of $h[0,0,1]$ defined by the formula $\phi(l)=' 1 \leq l \leq k$ ' (so that any specialization of $T_{k}$ is just a set of $k$ elements), and let $D_{k}$ be the set of all functions from $T_{k}$ to the set of two elements $\{ \pm 1\}$. Let $B_{\mathfrak{g}, r}$ be the subassignment

$$
B_{\mathfrak{g}, r}:=\bigsqcup_{k=1}^{n_{G}} A_{k, \mathfrak{g}, r} \times D_{k},
$$


where $A_{k, \mathfrak{g}, r}$ is as in Lemma 4.3 and $n_{G}$ is as in $\$ 1$. Note that some terms in the disjoint union in (5) may be empty, and that $B_{\mathfrak{g}, r}$ is equipped with a natural projection $\pi_{r}$ to $S_{\mathfrak{g}, r}$.

Remark 4.5. Alternatively, one can make the space parameterizing the orbital integrals from the spaces $S_{\mathfrak{g}, \mathfrak{h}, r}$ constructed in [14, where $\mathfrak{h}$ ranges over endoscopic Lie algebras for $\mathfrak{g}$. Observe also that it follows from 14 that the subassignment $\mathfrak{g}(r)$ of all restricted elements of depth $r$ is definable.

Proposition 4.6. There exists a morphism of definable subassignments $\nu_{r}: \mathfrak{g}(r) \rightarrow$ $B_{\mathfrak{g}, r}$, and there exists $M>0$ such that for every field $\mathbb{K} \in \mathcal{F}_{\mathbb{Z}, M}, \nu_{r}$ specializes to a surjective map $\nu_{r, \mathbb{K}}: \mathfrak{g}(r, \mathbb{K}) \rightarrow B_{\mathfrak{g}, r}\left(\mathbb{K}_{\mathbb{K}}\right)$ such that:

(1) The fibres of the map $\nu_{r}$ are exactly the thickened orbits: for every $y \in$ $B_{\mathfrak{g}, r}\left(\mathbb{k}_{\mathbb{K}}\right)$, the set $\nu_{r, \mathbb{K}}^{-1}(y)$ is a thickened orbit (see Definition 2.5),

$$
\nu_{r, \mathbb{K}}^{-1}(y)=\mathcal{O}_{r}\left(X_{y}\right)
$$

whenever $X_{y} \in \mathfrak{g}(r, \mathbb{K})$ satisfies $\nu_{r, \mathbb{K}}\left(X_{y}\right)=y$. In particular, if $[X]_{r}=$ $\left[X^{\prime}\right]_{r}$, then $\pi_{r} \circ \nu_{r, \mathbb{K}}(X)=\pi_{r} \circ \nu_{r, \mathbb{K}}\left(X^{\prime}\right)$, and $\nu_{r, \mathbb{K}}(X)=\nu_{r, \mathbb{K}}\left(X^{\prime}\right)$ if and only if $X^{\prime} \in \mathcal{O}_{r}(X)$.

(2) The restriction to $\mathcal{H}_{r}(\mathfrak{g}(\mathbb{K}))$ of the orbital integral of $X \in \mathfrak{g}(r, \mathbb{K})$ is completely determined by $\nu_{r, \mathbb{K}}(X)$. That is,

$$
\nu_{r, \mathbb{K}}(X)=\nu_{r, \mathbb{K}}\left(X^{\prime}\right) \Longleftrightarrow \forall f \in \mathcal{H}_{r}(\mathfrak{g}(\mathbb{K})), \mu_{X}(f)=\mu_{X^{\prime}}(f) .
$$

We denote the corresponding (restricted) orbital integral by $\mu_{y}$, where $y=$ $\nu_{r, \mathbb{K}}(X)$ (so this notation is well defined only for fields $\mathbb{K}$ of sufficiently large residue characteristic).

(3) There exists a positive constructible motivic function $Q_{\mathfrak{g}, r}(y)$ on $B_{\mathfrak{g}, r}$, and for every constructible motivic test function $f$ such that the Fourier transform of $f_{\mathbb{K}}$ is supported by $\mathfrak{g}(\mathbb{K})_{-r}$, there exists a constructible motivic function $\Phi^{f}$ on the definable subassignment $B_{\mathfrak{g}, r}$, such that for every field $\mathbb{K} \in \mathcal{F}_{M+M_{f}}$ (where $M_{f}$ might depend on $f$ ) we have

$$
\mu_{y}\left(f_{\mathbb{K}}\right)=\frac{\Phi_{\mathbb{K}}^{f}(y)}{Q_{\mathfrak{g}, r, \mathbb{K}}(y)},
$$

for every $y \in B_{\mathfrak{g}, r}\left(\mathbb{k}_{\mathbb{K}}\right)$, where $\mu_{y}\left(f_{\mathbb{K}}\right)$ stands for the value of the orbital integral of $f_{\mathbb{K}}$ along the orbit of $X_{y}$ for any $X_{y}$ such that $\nu_{r, \mathbb{K}}\left(X_{y}\right)=y$.

Proof. Let $\mathbb{K}$ be a local field, and let us take an element $X \in \mathfrak{g}(r, \mathbb{K})$. Let $\left(I, I^{*},\left(F_{i} / F_{i}^{\#}\right),\left(a_{i}\right),\left(c_{i}\right)\right)$ be the datum attached to $X$. Recall that $[X]_{r}=\mathcal{O}_{r}^{\text {st }}(X)$ (see Lemma 2.6) is a union of stable orbits. Let $x \in S_{\mathfrak{g}, r}\left(\mathbb{R}_{\mathbb{K}}\right)$ be the point that corresponds to the class $[X]_{r}$, according to [14, Theorem 4.4].

We need to choose a Kostant section (a map from the set of stable adjoint orbits in $\mathfrak{g}^{\text {reg }}(\mathbb{K})$ to the set of (rational) adjoint orbits in $\mathfrak{g}^{\text {reg }}(\mathbb{K})$ ). This map is associated with a choice of a nilpotent element $N \in \mathfrak{g}(\mathbb{K})$ and constructed by means of $\mathfrak{s l}_{2}$ triples. We need an explicit construction in Denef-Pas language, so we write $\widetilde{N}_{n}$ for the $n \times n$ matrix with 1's on the superdiagonal and 0's elsewhere, and then put

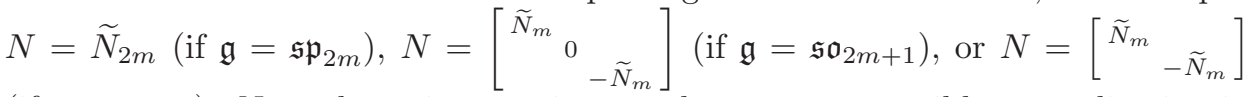
(if $\mathfrak{g}=\mathfrak{s o}_{2 m}$ ). Note that, since we just need some constructible normalization in what follows, we do not need to deal with the question of whether our construction 
depends on the choice of $N$ : as long as we always make the same choice of $N$, we will get the same bijection between the points of $B_{\mathfrak{g}, r}\left(\mathbb{k}_{\mathbb{K}}\right)$ and the equivalence classes of elements. As explained in [32, Section 2.4], the value of the Kostant section map at $X$ is obtained by intersecting the stable orbit of $X$ with a certain subvariety of $\mathfrak{g}$. We will refer to this subvariety as the Kostant section, as well.

Let $X_{0}$ be the element of the intersection of our Kostant section with the stable orbit that corresponds to the point $x$ (i.e., the stable orbit of $X$ ), and let $\left(\tilde{I}, \tilde{I}^{*},\left(\tilde{F}_{i} / \tilde{F}_{i}^{\#}\right),\left(\tilde{a}_{i}\right),\left(\tilde{c}_{i}\right)\right)$ be the Waldspurger's datum attached to $X_{0}$. Note that $\tilde{I}=I$ and $\tilde{I}^{*}=I^{*}$, that is, that the $I$ and $I^{*}$ pieces of the data for $X$ and $X_{0}$ are the same, since $X$ and $X_{0}$ are stably conjugate. We also may, and hence do, identify, for a fixed $i \in I$, the fields $F_{i}^{\#}$ and $\tilde{F}_{i}^{\#}$, and the algebras $F_{i}$ and $\tilde{F}_{i}$, corresponding to $X$ and $X_{0}$; see Proposition 4.1](2). For $i \in I^{*}$, let $\epsilon_{i}:=\operatorname{sgn}_{F_{i} / F_{i}^{\#}}\left(c_{i} \tilde{c}_{i}^{-1}\right)$, where $\operatorname{sgn}_{F_{i} / F_{i}^{\#}}$ is the sign character of $F_{i}^{\#}$ (this definition makes sense, since the element $c_{i} \tilde{c}_{i}^{-1}$ is stable under the involution $\tau_{i}$ of $F_{i}$, and therefore lies in $F_{i}^{\#}$ ).

Finally, let us define $\nu_{r, \mathbb{K}}(X)$ as

$$
\nu_{r, \mathbb{K}}(X)=\left(x,\left(\epsilon_{i}\right)_{i \in I^{*}(X)}\right) \in B_{\mathfrak{g}, r}\left(\mathbb{k}_{\mathbb{K}}\right) .
$$

Part (1). First, we prove that for a fixed field $\mathbb{K}$, the map $\nu_{r, \mathbb{K}}$ is well defined, and that its fibres are thickened orbits. Next, we will prove that $\nu_{r}$ is actually a morphism of definable subassignments.

We need to show that if $\nu_{r, \mathbb{K}}(X)=y=\left(x,\left(\epsilon_{i}\right)\right) \in B_{\mathfrak{g}, r}\left(\mathbb{k}_{\mathbb{K}}\right)$, then

$$
\nu_{r, \mathbb{K}}^{-1}(y)=\mathcal{O}_{r}(X) .
$$

As above, we denote by $\left(I, I^{*},\left(F_{i} / F_{i}^{\#}\right),\left(a_{i}\right),\left(c_{i}\right)\right)$ the Waldspurger's datum corresponding to $X$. Suppose that $Y \in \nu_{r, \mathbb{K}}^{-1}(y)$ has Waldspurger's datum

$\left(I^{\prime}, I^{* \prime},\left(F_{i}^{\prime} / F_{i}^{\prime \#}\right),\left(a_{i}^{\prime}\right),\left(c_{i}^{\prime}\right)\right)$. By the definition of $\nu_{r, \mathbb{K}}$, we have that $[Y]_{r}=[X]_{r}$, so, by Proposition 4.1 we may, and do, assume that $I=I^{\prime}, I^{*}=I^{* \prime}$, and, for all $i \in I, F_{i}^{\#}=F_{i}^{\prime \#}, F_{i}=F_{i}^{\prime}$ and the elements $a_{i}$ and $a_{i}^{\prime}$ have the same valuation and angular component. We claim that $\operatorname{sgn}_{F_{i} / F_{i}^{\#}}\left(c_{i} c_{i}^{\prime-1}\right)=1$.

Let us prove this claim. Let $\left(I, I^{*},\left(F_{i} / F_{i}^{\#}\right),\left(\tilde{a}_{i}^{\prime}\right),\left(\tilde{c}_{i}^{\prime}\right)\right)$ be the Waldspurger's datum that corresponds to the element $Y_{0}$ that is stably conjugate to $Y$ and lies in the Kostant section. It follows from the construction of the Kostant section (see, e.g. [32, Section 2.4]) combined with the proof of [14, Theorem 4.6] that we can assume that $\operatorname{sgn}_{F_{i} / F_{i}^{\#}}\left(\tilde{c}_{i}^{-1} \tilde{c}_{i}^{\prime}\right)=1$. Indeed, by the proof of [14, Theorem 4.6], for each element $X^{\prime \prime}$ in the stable orbit of $X$ there exists a unique element $Y^{\prime \prime}$ in the stable orbit of $Y$, such that $X^{\prime \prime}-Y^{\prime \prime}$ lies in $\mathfrak{g}(\mathbb{K})_{r^{+}}$, and this is the element that satisfies $\operatorname{sgn}_{F_{i} / F_{i}^{\#}}\left(c_{i}\left(X^{\prime \prime}\right) c_{i}\left(Y^{\prime \prime}\right)^{-1}\right)=1$ (where $c_{i}\left(X^{\prime \prime}\right)$, respectively, $c_{i}\left(Y^{\prime \prime}\right)$ stands for the $c$-part of the Waldspurger data for these elements). In particular, there is exactly one such element for $X_{0}$. Since the Kostant section is continuous by construction, this element must be the element $Y_{0}$ that also lies in the Kostant section. Finally, by definition of $\nu_{r, \mathbb{K}}$, we have

$$
\operatorname{sgn}_{F_{i} / F_{i}^{\#}}\left(c_{i} \tilde{c}_{i}^{-1}\right)=\operatorname{sgn}_{F_{i} / F_{i}^{\#}}\left(c_{i}^{\prime} \tilde{c}_{i}^{\prime-1}\right),
$$

so that

$$
\operatorname{sgn}_{F_{i} / F_{i}^{\#}}\left(c_{i} c_{i}^{\prime-1}\right)=\operatorname{sgn}_{F_{i} / F_{i}^{\#}}\left(\tilde{c}_{i} \tilde{c}_{i}^{\prime-1}\right)=1 .
$$


To summarize, we have shown that if $\nu_{r, \mathbb{K}}(Y)=\nu_{r, \mathbb{K}}(X)$, then the elements $X$ and $Y$ have the data $\left(I, I^{*},\left(F_{i} / F_{i}^{\#}\right),\left(a_{i}\right),\left(c_{i}\right)\right)$ and $\left(I, I^{*},\left(F_{i} / F_{i}^{\#}\right),\left(a_{i}^{\prime}\right),\left(c_{i}^{\prime}\right)\right)$, respectively; and, for all $i \in I$, the elements $a_{i}$ and $a_{i}^{\prime}$ have the same valuation and angular component; and $\operatorname{sgn}_{F_{i} / F_{i}^{\#}}\left(c_{i} c_{i}^{\prime-1}\right)=1$.

It is shown in the proof of [14, Theorem 4.6] that the conditions on $I,\left(F_{i} / F_{i}^{\#}\right)$, and $\left(a_{i}\right)$ are equivalent to the existence of topologically unipotent elements $u_{i} \in F_{i}$ such that $\lambda_{i}(X)=u_{i} \lambda_{i}(Y)$, where $\lambda_{i}$ are the appropriately labelled eigenvalues. This, in turn, combined with the conditions $\operatorname{sgn}_{F_{i} / F_{i}^{\#}}\left(c_{i} c_{i}^{\prime-1}\right)=1$, is equivalent to the statement that there exists a rational conjugate $Y^{\prime}$ of $Y$, such that $X-Y^{\prime}$ lies in $\mathfrak{g}(\mathbb{K})_{r^{+}}$(note that we have shown in Remark 2.2 that the constant that is used to relate depth and slope in [14 is, in fact, 1 in all of our cases).

Finally, note that for $x \in S_{\mathfrak{g}, r}\left(\mathbb{k}_{\mathbb{K}}\right)$, upon taking the union over all $y \in B_{\mathfrak{g}, r}\left(\mathbb{k}_{\mathbb{K}}\right)$ satisfying $\pi_{r}(y)=x$ on both sides of ([6), we get the assertion of Lemma 2.6]

We have established the properties of the map $\nu_{r, \mathbb{K}}$ with $\mathbb{K}$ fixed. Now we need to show that $\nu_{r}$ is a definable map. That means we need to show that the graph of $\nu_{r}$ is definable. We will show that the fibres of $\nu_{r}$ form a definable family of definable sets, and that will imply the definability of the graph.

As we have just shown, given a field $\mathbb{K}$ with sufficiently large residue characteristic, we can unambiguously use the notation $\mathcal{O}_{r}(y):=\nu_{r, \mathbb{K}}^{-1}(y)$, for $y \in B_{\mathfrak{g}, r}\left(\mathbb{k}_{\mathbb{K}}\right)$. To prove that $\mathcal{O}_{r}(y)$ is definable (i.e., that it is obtained from a definable subassignment by specialization), we use the techniques from [16. Recall the notation, and a few facts proved there that are relevant for the present situation.

(1) Each $F_{i}^{\#}$ and $F_{i}$ is a $\mathbb{K}$-vector space. As in [40, the elements $\left(c_{i}\right)$ determine a pairing $q_{W}$ on the vector space $W:=\bigoplus_{i \in I} F_{i}$ such that $\left(V, q_{V}\right)$ and $\left(W, q_{W}\right)$ are isomorphic as quadratic spaces (where $V$ is the quadratic space naturally associated to our group $G$ ). Let $\phi: W \rightarrow V$ be such an isomorphism, and let $\phi_{*}: \operatorname{End}(W) \rightarrow \operatorname{End}(V)$ be the isomorphism induced by $\phi$.

Let $L_{i}: F_{i} \rightarrow \operatorname{End}\left(F_{i}\right)$ be the linear map that takes $w \in F_{i}$ to the linear operator that acts by left multiplication by $w$ on $F_{i}$. Let $L: W \rightarrow$ $\operatorname{End}(W)$ be the direct sum of the maps $L_{i}$. Thus, if the space $\left(W, q_{W}\right)$ was constructed from Waldspurger's datum for a regular semisimple element $X$, then for $w \in W$, its image $\phi_{*} \circ L(w)$ can be thought of as an element of $C(X)$ - the centralizer of $X$.

(2) Let $X$ be an $n \times n$-matrix with distinct eigenvalues $\left\{\lambda_{j}\right\}$, and with the characteristic polynomial $P_{X}(\lambda)=\prod_{j}\left(\lambda-\lambda_{j}\right)$. Let $P^{(j)}(\lambda)$ be the polynomial $P_{X}$ divided by the factor $\left(\lambda-\lambda_{j}\right)$. Then the projection operator onto the $\lambda_{j}$-eigenspace can be written as $P^{(j)}(X) / P^{(j)}\left(\lambda_{j}\right)$. More generally, if we write $P_{X}=f \tilde{f}$ as a product of two monic polynomials $f$ and $\tilde{f}$, then there exists a polynomial $\Pi=\Pi(\lambda, f, \tilde{f})$ (see [16, §1.7]) that, when evaluated at $X$, defines the projection operator onto the direct sum of the eigenspaces of $X$ that correspond to the roots of $f$. The key fact here is that the coefficients of $\Pi$ are polynomial expressions in the matrix entries $x_{i j}$ of $X$, and in the coefficients of $f$ and $\tilde{f}$.

(3) [16, Lemma 35] Let $z_{i} \in F_{i}^{\#}$ be an arbitrary element. Then the following conditions are equivalent:

(a) $\operatorname{sgn}_{F_{i} / F_{i}^{\#}}\left(z_{i}\right)=1$. 
(b) Let $w$ be an arbitrary element of $W$ such that $w_{i}=z_{i}$. Let $P$ be the projector from $C(X)$ onto $\phi_{*} \circ L\left(F_{i}\right)$ as above. (Essentially, it is a projector to the $\lambda_{i}$-eigenspace of $X$.) Then there exists $X_{1} \in C(X)$ such that $P X_{1} \tau\left(X_{1}\right)=P\left(\phi_{*} \circ L\right)(w)$. Here $\tau$ is the involution on $C(X)$ defined by $\tau(g)=\left(J^{-1}\right)^{t} g J$, where $J$ is the matrix from the definition of the group $G$ (see (11) and (2) on page 534).

Now we are ready to finish the proof that the sets $\mathcal{O}_{r}(y)$ are definable for $y \in$ $B_{\mathfrak{g}, r}\left(\mathbb{k}_{\mathbb{K}}\right)$, and depend on $y$ in a definable way. Let $y \in B_{\mathfrak{g}, r}\left(\mathbb{k}_{\mathbb{K}}\right)$. Then $y=$ $\left(x,\left(\epsilon_{i}\right)_{i \in I^{*}(x)}\right)$, where $x \in S_{\mathfrak{g}, r}\left(\mathbb{k}_{\mathbb{K}}\right)$ and $\epsilon_{i}= \pm 1$.

By the construction of the subassignment $B_{\mathfrak{g}, r}$, the number of indices $i$ such that $\epsilon_{i}=1$ equals the cardinality $\left|I^{*}(x)\right|$ by Lemma 4.3. Let $X \in \mathcal{O}_{r}(y)$. We have the element $X_{0}$ in the stable conjugacy class of $X$ that lies in the Kostant section. We can think of the entries of the matrix $X_{0}$ as terms in Denef-Pas language (see [12]). Now, let $\left(\tilde{c}_{i}\right)$ be the $\left(c_{i}\right)$-part of Waldspurger's datum that corresponds to $X_{0}$, and let $c_{0}=\phi_{*} \circ L\left(\tilde{c}_{i}\right)$. Note that all of this can be written as one logical formula in the notation of [16]:

$$
\exists c_{0} \in C\left(X_{0}\right) \text { trace-form }\left(X_{0}, c_{0}\right)
$$

(see Definition 24 loc. cit. for the definition of the term "trace-form", and Remark 34 loc. cit. for this statement). Similarly, for our element $X$, we have an element $c \in C(X)$ satisfying the same condition with $X$ replacing $X_{0}$. The condition that $X \in \mathcal{O}_{r}(y)$ can be restated as

$$
\operatorname{sgn}_{F_{i} / F_{i}^{\#}}\left(c_{i} \tilde{c}_{i}^{-1}\right)=\epsilon_{i} \forall i \in I^{*} .
$$

By Lemma 35 loc. cit. (quoted as item (3) above), this condition can be expressed by a formula in Denef-Pas language, whose variables include $x$ (interpreted as a tuple of variables of the residue field), and $\epsilon_{i}$. In particular, the dependence on $y$ is definable. 2.8

Part (2). This follows immediately from Part (1), combined with Proposition

Part (3). Finally, to prove the last statement, we just need to modify slightly the argument of [14, Lemma 6.2]. For a point $y \in B_{\mathfrak{g}, r}\left(\mathbb{k}_{\mathbb{K}}\right)$, let $\mathcal{O}_{r}(y)=\nu_{r, \mathbb{K}}^{-1}(y)$, as above. It is an open subset of $\mathfrak{g}(r, \mathbb{K})$. Let $X_{y}$ be an element in $\nu_{r, \mathbb{K}}^{-1}(y)$, and let $\mathfrak{t}$ be the Cartan subalgebra containing $X_{y}$. We think of $\mathcal{O}_{r}(y)$ as a neighbourhood of the orbit of $X_{y}$, and recall that

$$
\mathcal{O}_{r}(y)=\bigcup_{Y \in \mathfrak{t}(\mathbb{K})_{r+}} \mathcal{O}\left(X_{y}+Y\right) .
$$

Recall the Weyl integration formula:

$$
\begin{aligned}
\int_{\mathfrak{g}(\mathbb{K})} f(X) d X= & \sum_{T^{\prime}}\left|W\left(G(\mathbb{K}), T^{\prime}(\mathbb{K})\right)\right|^{-1} \\
& \times \int_{\mathfrak{t}^{\prime}(\mathbb{K})}\left|D^{\mathfrak{g}}(X)\right| \int_{G(\mathbb{K}) / T^{\prime}(\mathbb{K})} f(\operatorname{Ad} x X) d x d X,
\end{aligned}
$$

where the summation is over the $G(\mathbb{K})$-conjugacy classes of maximal tori $T^{\prime}$ in $G$. Let $f \in \mathcal{H}_{r}(\mathfrak{g}(\mathbb{K}))$ be a definable test function (by which we mean that it is a specialization of some constructible motivic function), and let $f_{y}$ be the characteristic function of the set $\mathcal{O}_{r}(y)$. Let us apply the Weyl integration formula to the function $f f_{y}$. It follows from the proof of [14, Theorem 4.6] that, if two restricted 
elements $X, X^{\prime} \in \mathfrak{g}(r, \mathbb{K})$ satisfy $\nu_{r, \mathbb{K}}(X)=\nu_{r, \mathbb{K}}\left(X^{\prime}\right)$, then their centralizers are $G(\mathbb{K})$-conjugate; cf. Proposition 4.1(1). Therefore, since $f_{y}$ vanishes outside $\mathcal{O}_{r}(y)$, the right-hand side of (7) will have only one non-zero summand, corresponding to $T=C_{G}\left(X_{y}\right)$. Then the right-hand side equals

$$
|W(G(\mathbb{K}), T(\mathbb{K}))|^{-1} \int_{X_{y}+\mathfrak{t}(\mathbb{K})_{r+}}\left|D^{\mathfrak{g}}(X)\right| \int_{G(\mathbb{K}) / T(\mathbb{K})} f\left(\operatorname{Ad}_{x} X\right) d x d X .
$$

Since the orbital integral

$$
\int_{G(\mathbb{K}) / T(\mathbb{K})} f\left(\operatorname{Ad}_{x} X\right) d x
$$

as a function of $X$, is constant on the $\operatorname{coset} X_{y}+\mathfrak{t}(\mathbb{K})_{r^{+}}$by Proposition 2.8, we get

$$
\int_{\mathfrak{g}(\mathbb{K})} f f_{y}(X) d X=|W(G(\mathbb{K}), T(\mathbb{K}))|^{-1}\left|D^{\mathfrak{g}}\left(X_{y}\right)\right| \mu_{X_{y}}(f) \operatorname{vol}^{*}\left(X_{y}+\mathfrak{t}(\mathbb{K})_{r^{+}}\right),
$$

where $\operatorname{vol}^{*}$ is the volume on $\mathfrak{t}(\mathbb{K})$, (see [14, Section 6] for the discussion of the appropriate normalization for $\left.\operatorname{vol}^{*}\right)$. The factor $|W(G(\mathbb{K}), T(\mathbb{K}))|^{-1}\left|D^{\mathfrak{g}}\left(X_{y}\right)\right|$ is constructible by [14, Lemma 6.1 and (6.1.3)]. The factor $\operatorname{vol}^{*}\left(X_{y}+\mathfrak{t}(\mathbb{K})_{r^{+}}\right)$is a specialization of a constructible motivic function of $y$ by [11, Theorem 10.1.1], since the set $X_{y}+\mathfrak{t}(\mathbb{K})_{r+}$ depends on $y$ in a definable way. We denote by $Q_{\mathfrak{g}, r}$ the element of $\mathcal{C}\left(B_{\mathfrak{g}, r}\right)$ that specializes to

$$
|W(G(\mathbb{K}), T(\mathbb{K}))|^{-1}\left|D^{\mathfrak{g}}\left(X_{y}\right)\right| \operatorname{vol}^{*}\left(X_{y}+\mathfrak{t}(\mathbb{K})_{r^{+}}\right) .
$$

The left-hand side of (8) is $\int_{\mathcal{O}_{r}(y)} f(X) d X$. Thus, the equality (8) can be rewritten as:

$$
\mu_{X_{y}}(f)=\frac{|W(G(\mathbb{K}), T(\mathbb{K}))|}{\left|D^{\mathfrak{g}}\left(X_{y}\right)\right| \operatorname{vol}^{*}\left(X_{y}+\mathfrak{t}(\mathbb{K})_{r^{+}}\right)} \int_{\mathcal{O}_{r}(y)} f(X) d X=\frac{1}{Q_{\mathfrak{g}, r, \mathbb{K}}(y)} \int_{\mathcal{O}_{r}(y)} f(X) d X .
$$

By Part (1), the set $\mathcal{O}_{r}(y)$ is definable and depends on $y$ in a definable way. More precisely, it is a specialization of the fibre over $y$ of an an element of $\operatorname{Def}_{B_{\mathfrak{g}, r}}$; therefore, the left-hand side of (8) is a specialization of a constructible motivic function of $y$, by [11, Theorem 10.1.1].

The last claim now follows from [11, Theorem 10.1.1] together with the results on specialization of motivic integrals, [9, Theorems 6.9 and 7.3].

\subsection{Proof of Theorem 2.21,}

4.3.1. Part (1). Let $\mathbb{K}$ be any local field of residue characteristic bigger than $2, \pi$ a restricted representation of $G(\mathbb{K})$ of minimal depth $r$, and $\Psi$ the associated cuspidal datum (see Section 2.6).

Recall from Theorem 2.18 that there is some element $\Gamma_{\Psi} \in \mathfrak{g}(-r, \mathbb{K})$ such that, for every test function $f$ with support contained in $G(\mathbb{K})_{r}^{\text {reg }}$, we have

$$
\frac{1}{\operatorname{deg}(\pi)} \Theta_{\pi}(f)=\hat{\mu}_{\Gamma_{\Psi}}\left(f \circ \mathrm{e}^{-1}\right)=\mu_{\Gamma_{\Psi}}\left(\widehat{f \circ \mathrm{e}^{-1}}\right) .
$$

By Lemma 2.10, $\widehat{f \circ \mathrm{e}^{-1}}$ lies in the space $\mathcal{H}_{-r}(\mathfrak{g}(\mathbb{K}))$. By Proposition 4.6(1), there exists $M>0$ such that if the residue characteristic of $\mathbb{K}$ is greater than $M$, the element $\Gamma_{\Psi}$ projects to a point $y_{\Psi} \in B_{\mathfrak{g},-r}\left(\mathbb{K}_{\mathbb{K}}\right)$. By Proposition $4.6(3)$, the restriction of the distribution $\mu_{\Gamma_{\Psi}}$ to the space $\mathcal{H}_{-r}(\mathfrak{g}(\mathbb{K}))$ depends only on the point $y_{\Psi}$. Hence, the point $y_{\Psi} \in B_{\mathfrak{g},-r}\left(\mathbb{k}_{\mathbb{K}}\right)$ determines the restriction of $\Theta_{\pi}$ to $G(\mathbb{K})_{r}^{\mathrm{reg}}$. This 
argument shows that for fields $\mathbb{K}$ with sufficiently large residue characteristic, the map

$$
\pi_{\Psi} \mapsto \nu_{-r, \mathbb{K}}\left(\Gamma_{\Psi}\right)
$$

is a well-defined one-to-one map from the set of $r$-equivalence classes of restricted representations of $G(\mathbb{K})$ of minimal depth $r$ to $B_{\mathfrak{g},-r}\left(\mathbb{K}_{\mathbb{K}}\right)$.

4.3.2. Part (2). This follows immediately from the equality (10) and Proposition $4.6(4)$.

4.3.3. Part (3). We have proved that the sets $\mathcal{O}_{-r}(y)$ form a definable family of definable sets parameterized by a variable $y$ running over the definable subassignment $B_{\mathfrak{g},-r}$. Therefore, given a definable family of definable functions $\left\{f_{a}\right\}$ (parameterized by $a$ in some subassignment $A)$, the expression $F(y, a):=\int_{\mathcal{O}_{-r}(y)} \widehat{f_{a} \circ \mathrm{e}^{-1}}$ is a constructible motivic exponential function of $y$ and $a$ by [10, Theorem 4.1.1]. Then, by the specialization principle [10, Theorem 9.1.5], there exists a constant $M^{\prime}$ (that depends on the family $\left\{f_{a}\right\}$ and on $\mathfrak{g}$ and $r$ ), such that for all fields $\mathbb{K} \in \mathcal{F}_{\mathbb{Z}, M^{\prime}}$, the motivic integral $\int_{\mathcal{O}_{-r}(y)} \widehat{f_{a} \circ \mathrm{e}^{-1}}$ specializes to the $p$-adic integral $\int_{\mathcal{O}_{-r}\left(X_{y}\right)} \widehat{f_{a, \mathbb{K}} \circ \mathrm{e}^{-1}}$, where $X_{y} \in \mathfrak{g}(\mathbb{K})$ is any element in $\nu_{-r, \mathbb{K}}^{-1}(y)$ (recall that the subassignment $\mathcal{O}_{-r}(y)$ specializes to the fibre over $y$ of the map $\nu_{-r, \mathbb{K}}: \mathfrak{g}(-r, \mathbb{K}) \rightarrow$ $\left.B_{\mathfrak{g},-r}\left(\mathbb{K}_{\mathbb{K}}\right)\right)$.

Further, let $M^{\prime \prime}$ be the constant such that for all $\mathbb{K} \in \mathcal{F}_{\mathbb{Z}, M^{\prime \prime}}$, the specialization principle holds for the constructible motivic function $Q_{\mathfrak{g},-r} \in \mathcal{C}\left(B_{\mathfrak{g},-r}\right)$ from Proposition 4.6, Part (3). Note that $M^{\prime \prime}$ depends only on $\mathfrak{g}$ and $r$.

Let $M_{0}$ be the maximum of the constant $M$ from Part (1) and $M^{\prime}, M^{\prime \prime}$ defined above. Let $\mathbb{K} \in \mathcal{F}_{\mathbb{Z}, M_{0}}$, and let $\pi$ be a restricted representation of $G(\mathbb{K})$ of minimal depth $r$, as above. Let $\Gamma_{\Psi} \in \mathfrak{g}(-r, \mathbb{K})$ be the element corresponding to $\pi$ (see Definition 2.14), and let $y \in B_{\mathfrak{g},-r}\left(\mathbb{K}_{\mathbb{K}}\right)$ be the image of $\Gamma_{\Psi}$ under $\nu_{-r, \mathbb{K}}$. Then, using the notation of the proof of Proposition 4.6 we can write $\Gamma_{\Psi}=X_{y}$. Let $T$ be the centralizer of $X_{y}$. We have (using the equality (9)):

$$
\frac{1}{\operatorname{deg}(\pi)} \Theta_{\pi}\left(f_{a}\right)=\mu_{\Gamma_{\Psi}}\left(\widehat{f_{a} \circ \mathrm{e}^{-1}}\right)=\frac{1}{Q_{\mathfrak{g},-r, \mathbb{K}}(y)} F_{\mathbb{K}}(y, a) .
$$

Thus, we have shown that $\frac{Q_{\mathfrak{g},-r, \mathbb{K}}(y)}{\operatorname{deg}(\pi)} \Theta_{\pi}\left(f_{a}\right)$ is a constructible exponential function of $a$. Since $\frac{Q_{\mathfrak{g},-r, \mathbb{K}}(y)}{\operatorname{deg}(\pi)}$ is a positive rational number that does not depend on $a$, this completes the proof.

Remark 4.7. Rephrasing Part (1) of the theorem we just proved, we would like to emphasize that we can reconstruct (in an algorithmically computable way) the values of the distribution character, at least at a family of definable test functions supported near the identity and satisfying the conditions from Definition 2.20. from a point $y \in B_{\mathfrak{g},-r}\left(\mathbb{k}_{\mathbb{K}}\right)$. Indeed, starting with a point $y \in B_{\mathfrak{g},-r}\left(\mathbb{K}_{\mathbb{K}}\right)$, we can construct the fibre $\mathfrak{g}(-r)_{y, \mathbb{K}}=\nu_{-r, \mathbb{K}}^{-1}(y)$ (which is a thickened orbit) of the subassignment $\mathfrak{g}(-r)$ from Proposition 4.6(1), for all local fields $\mathbb{K}$ with residue characteristic bigger than a constant, which we will denote by $M_{\mathfrak{g}, r}$, that depends only on $\mathfrak{g}$ and $r$. Now, let us consider the family $\xi_{a, n}$ of Section 3.2.1. By Lemma 3.5. there exists a constant $M_{\xi, \mathfrak{g}, r}>0$, such that for $\mathbb{K} \in \mathcal{F}_{\mathbb{Z}, M_{\xi, \mathfrak{g}, r}}$, the motivic integral $\int_{\mathcal{O}_{-r}} \xi_{a, n}$ specializes to the integral $\int_{\nu_{-r, \mathbb{K}}^{-1}(y)} \xi_{a, n, \mathbb{K}} d g$, where the latter integral is with respect to the Haar measure on $G(\mathbb{K})$. Here we apply Lemma 3.5 to 
the family $\left\{f_{\alpha}\right\}$ that consists of the characteristic functions of the thickened orbits $\nu_{-r, \mathbb{K}}^{-1}(y)$, indexed by the points $(y, \mathbb{K})$ of the definable subassignment $B_{\mathfrak{g},-r}$. The constant $M_{\xi, \mathfrak{g}, r}$ depends on $M_{\xi}$ and on the formulas defining the subassignment $B_{\mathfrak{g},-r}$, which, in turn, depend only on $G$ and $r$. For the fields of characteristic zero, where we know that the distribution character is a continuous distribution, this information is equivalent to knowing the restriction of the distribution character to $G(\mathbb{K})_{r}$.

4.4. Proof of Theorem 2.23 in the characteristic-zero case. We will use J. Korman's theorem on the local constancy of characters 31, which we quote here. That paper assumes that characteristic of $\mathbb{K}$ is zero, so we will assume that throughout this section.

Let $\gamma \in G(\mathbb{K})^{\text {reg }}$ be a compact element, and assume that the connected component of the centralizer of $\gamma$ is a torus that splits over a tamely ramified extension $E$ of $\mathbb{K}$.

Let $T=C_{G}(\gamma)^{\circ}$. Then the regular depth of $\gamma$ (see [31, Definition 1.1] or [5. Definition 4.1], where the term singular depth is used instead) is the rational number

$$
s(\gamma)=\max \left\{\operatorname{ord}_{\mathbb{K}}(\alpha(\gamma)-1) \mid \alpha \in \Phi(G, T)\right\} .
$$

Note that the following theorem does not require our strong hypotheses on the representation $\pi$.

Theorem 4.8 ([31, Theorem 4.1]). Let $\pi$ be an irreducible admissible representation and choose $r^{\prime}>\max \{s(\gamma), \mathrm{d}(\pi)\}$, where $\mathrm{d}(\pi)$ is the depth of the representation $\pi$. Then the distribution $\Theta_{\pi}$ is represented on the set ${ }^{G(\mathbb{K})}\left(\gamma T(\mathbb{K})_{r^{\prime}+s(\gamma)}\right)$ by a constant function.

In fact, this theorem follows from Corollary 11.9 of $[5$, (since, in the notation of that result, we have $\mathfrak{m}=\operatorname{Lie}(T)(\mathbb{K})$ in our case, so that $\mathcal{O}_{\mathfrak{m}}(0)=\{0\}$ ), which makes no assumption on the characteristic of the field $\mathbb{K}$. Hence, the argument presented in this section for the fields of characteristic zero works in positive characteristic as well. However, since part of the strength of motivic integration lies in the transfer between characteristic-zero and positive-characteristic cases, we decided to include the next section, in which we derive the positive-characteristic version of Theorem 2.23 from the characteristic-zero case.

Now let us assume that the residue characteristic $p$ is larger than $n=n_{G}$, in the notation of $\$ 1$ and that $\gamma \in G(\mathbb{K})_{r}^{\mathrm{reg}}$. Then, since $G$ embeds in $\mathrm{GL}_{n}$ and (in this setting) every torus in $\mathrm{GL}_{n}$ splits over a tame extension of $\mathbb{K}$, the condition that the centralizer of $\gamma$ splits over a tamely ramified extension is fulfilled automatically. Since $\gamma$ is compact, we can apply Theorem 4.8 .

Let $G_{0^{+}}^{\text {reg }}$ be the subassignment that specializes, for every $\mathbb{K} \in \mathcal{F}_{\mathbb{Z}, M}$, to the set of regular topologically unipotent elements $G(\mathbb{K})_{0^{+}}^{\text {reg }}$ (see 2.7 .1 ). Thus, the term

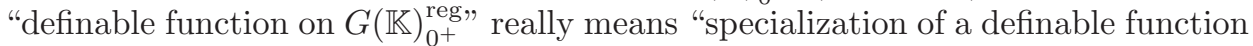
on $G_{0^{+}}^{\mathrm{reg}}$.

Lemma 4.9. There exists $M>0$, and a definable function $\phi(\gamma)$ on the set $G(\mathbb{K})_{0^{+}}^{\mathrm{reg}}=G_{0^{+}, \mathbb{K}}^{\mathrm{reeg}}$ that satisfies $\phi(\gamma) \geq s(\gamma)$ for all $\gamma \in G(\mathbb{K})_{0^{+}}^{\mathrm{reg}}$, for all $\mathbb{K} \in \mathcal{F}_{\mathbb{Z}, M}$.

Proof. Let $X=\mathrm{e}^{-1}(\gamma)$, a regular, semisimple, topologically nilpotent element in $\mathfrak{g}(\mathbb{K})$. Then $s(\gamma)=\max \left\{\operatorname{ord}_{\mathbb{K}}(d \alpha(X)) \mid \alpha \in \Phi(G, T)\right\}$. Note that $D^{\mathfrak{g}}(X)=$ 
$\operatorname{det} \operatorname{ad}(X)$ is obviously a definable function of $X$, and it has the expression

$$
D^{\mathfrak{g}}(X)=\operatorname{det} \operatorname{ad}(X)=\prod_{\alpha} d \alpha(X) .
$$

Therefore, since $d \alpha(X) \in \mathcal{O}_{\mathbb{K}}$ for every $\alpha \in \Phi(G, T)$, we have for all such $\alpha$ that

$$
\operatorname{ord}_{\mathbb{K}}(d \alpha(X)) \leq \operatorname{ord}_{\mathbb{K}} D^{\mathfrak{g}}(X) ;
$$

so we can take $\phi(\gamma)=\operatorname{ord}_{\mathbb{K}} D^{\mathfrak{g}}(X)$.

With some care to handle the case when $T$ is not split, we expect that we could show that $s(\gamma)$ itself is a definable function. However, for our purposes, this is not necessary.

Corollary 4.10. There exists $M>0$ and a definable subassignment $\mathcal{T}$ in the category $\operatorname{Def}_{G_{0^{+}}^{\text {reg }}}$, such that for every $\mathbb{K} \in \mathcal{F}_{\mathbb{Z}, M}$, and every $\gamma \in G(\mathbb{K})_{0^{+}}^{\mathrm{reg}}$, the fibre $\mathcal{T}_{\gamma, \mathbb{K}}$ is the set $\gamma T(\mathbb{K})_{r+2 \phi(\gamma)}$. The restriction of the character $\theta_{\pi}$ to each of the sets $\gamma T(\mathbb{K})_{r+2 \phi(\gamma)}$ is constant.

Remark 4.11. We observe that the corollary implies that the sets $G(\mathbb{K})\left(\gamma T(\mathbb{K})_{r+2 \phi(\gamma)}\right)$ can also be thought of as the fibres of a definable subassignment. However, we cannot tell whether the individual level sets $G(\mathbb{K})\left(\gamma T(\mathbb{K})_{r^{\prime}+s(\gamma)}\right)$ are definable (i.e., whether they are in $\left.\operatorname{Def}_{\mathbb{Q}}\right)$, a priori, there is no reason why we could eliminate the parameter $\gamma$, so this corollary does not automatically imply that $\theta_{\pi}$ is a constructible exponential function.

It follows from the above corollary that $G(\mathbb{K}) \mathcal{T}_{\gamma, \mathbb{K}}$ is a definable neighbourhood of the conjugacy class of $\gamma$ on which the character $\theta_{\pi}$ is constant. We would like to get a compact definable neighbourhood of $\gamma$, with the additional property that its volume is an invertible element in the ring of values of the motivic measure. First, we replace the neighbourhood ${ }^{G(\mathbb{K})} \mathcal{T}_{\gamma, \mathbb{K}}$ by something compact. Let $x$ be the hyperspecial vertex in $\mathcal{B}(G, \mathbb{K})$ such that $G(\mathbb{K})_{x, 0}=G\left(\mathcal{O}_{\mathbb{K}}\right)$, which is, clearly, definable. Then by the above corollary, there is a definable subassignment, which we will denote by $\mathcal{T}^{0}$, such that

$$
\mathcal{T}_{\gamma, \mathbb{K}}^{0}={ }^{G(\mathbb{K})_{x, 0}}\left(\gamma T(\mathbb{K})_{r+2 \phi(\gamma)}\right)
$$

for $\mathbb{K}$ of sufficiently large residue characteristic and $\gamma \in G(\mathbb{K})$. The set $\mathcal{T}_{\gamma, \mathbb{K}}^{0}$ is a compact, definable neighbourhood of $\gamma$ on which $\theta_{\pi}$ is constant. We have that $\operatorname{vol}\left(\mathcal{T}_{\gamma, \mathbb{K}}^{0}\right)$ is a constructible motivic function of $\gamma$, since it computes the volumes of the fibres of a definable subassignment. However, later we would like to divide another constructible motivic function by this volume function, and it might not be an invertible element in the ring of constructible motivic functions. The next lemma shows how to fix this by passing to a further subassignment.

Lemma 4.12. There exists a definable subassignment $U$ in $\operatorname{Def}_{G_{0^{+}}^{\text {reg }}}$, such that for every $\mathbb{K} \in \mathcal{F}_{\mathbb{Z}, M}$, for every $\gamma \in G(\mathbb{K})_{0^{+}}^{\mathrm{reg}}$, we have $U_{\gamma, \mathbb{K}} \subset \mathcal{T}_{\gamma, \mathbb{K}}^{0}$, and the motivic volumes of the fibres $U_{\gamma}$ are of the form $\mathbb{L}^{n(\gamma)}$, with $n(\gamma)$ a $\mathbb{Z}$-valued constructible function on $G_{0^{+}}^{\mathrm{reg}}$.

Proof. Note that $\mathcal{T}_{\gamma, \mathbb{K}}^{0}$ is an open subset of $G(\mathbb{K})$ that depends only on the parameter $\gamma$. Let $d$ stand for the dimension of $G$ (as an algebraic variety, which coincides with its dimension as a definable subassignment in the sense of [11, Section 3.1], as well as with the dimension of $G(\mathbb{K})$ as a $p$-adic manifold). We will call a subset of $\mathbb{A}^{d}(\mathbb{K})$ 
a ball if it is defined by a formula ord $\left(x_{i}-a_{i}\right) \geq r_{i}$ in the variables $\left(x_{1}, \ldots, x_{d}\right)$ for some element $a=\left(a_{i}\right)_{1 \leq i \leq d} \in \mathbb{A}^{d}(\mathbb{K})$, and for $r=\left(r_{i}\right)_{1 \leq i \leq d} \in \mathbb{Z}^{d}$. Notice that the volume of such a $p$-adic ball with respect to the Haar measure on $\mathbb{A}^{d}(\mathbb{K})$, normalized so that meas $\mathcal{O}_{\mathbb{K}}^{d}=1$, is of the form $q^{n}$, where the power $n \in \mathbb{Z}$ depends on $r$. Now, the idea is to make a subassignment $U$ such that $U_{\gamma, \mathbb{K}}$ is a ball (with respect to some suitable local coordinates around $\gamma$ ).

One convenient choice of coordinates on $G$ for the study of Haar measure comes from a big Bruhat cell. Let $T_{\text {spl }}$ be a maximal $\mathbb{K}$-split torus in $G, B$ a Borel subgroup containing $T_{\mathrm{spl}}, N$ its unipotent radical, $B^{-}$the opposite Borel subgroup (with respect to $T_{\mathrm{spl}}$ ), and $N^{-}$the opposite unipotent radical. The subvariety $\Omega:=N^{-} T_{\mathrm{spl}} N$ of $G$, which is a translate of the standard big cell in $G$, is isomorphic to the Cartesian product $N^{-} \times T_{\text {spl }} \times N$. The complement of $\Omega(\mathbb{K})$ in $G(\mathbb{K})$ has Haar measure 0 . As varieties, $N$ and $N^{-}$are isomorphic to affine spaces of the same dimension, and $T$ to an open subset of an affine space. Let $x_{1}, \ldots, x_{s}$ be coordinates on $N ; y_{1}, \ldots, y_{s}$ coordinates on $N^{-}$; and $s_{1}, \ldots, s_{r}$ coordinates on $T_{\text {spl }}$. With this choice of coordinates, the extension by 0 to $G(\mathbb{K})$ of the volume form

$$
\omega=d y_{1} \wedge \cdots \wedge d y_{s} \wedge d x_{1} \cdots \wedge d x_{s} \wedge \frac{d s_{1}}{s_{1}} \wedge \cdots \wedge \frac{d s_{r}}{s_{r}}
$$

on $\Omega(\mathbb{K})$ is a Haar measure (cf. [18]). To abbreviate, we will write $(x, y, s)$ for the coordinates, meaning the corresponding tuples.

For every $\gamma \in G(K)_{0^{+}}^{\mathrm{reg}} \cap \Omega(\mathbb{K})$, there exists a ball, (with respect to the coordinates $(x, y, s)$, in the sense of the definition above) that contains $\gamma$, and is contained in $\Omega(\mathbb{K}) \cap \mathcal{T}_{\gamma, \mathbb{K}}^{0}$, and such that $\left|s_{i}\right|$ is constant on this ball for $i=1, \ldots, r$. For every $\gamma$, there exists unique largest ball with these properties. We denote it by $C_{1}(\gamma)$. By the definition of the volume form $\omega$, the volume of this ball depends on $\gamma$ in a definable way, and is of the required form. We define the subassignment $U$ of $G_{0^{+}}^{\mathrm{reg}} \times \mathbb{A}^{d}$ in such a way that $U_{\gamma, \mathbb{K}}=C_{1}(\gamma)$ for $\gamma \in G(K)_{0^{+}}^{\mathrm{reg}} \cap \Omega(\mathbb{K})$.

Finally, there are finitely many translates of the set $\Omega(\mathbb{K})$ that cover $G(\mathbb{K})$. Let us denote them by $\Omega_{1}, \ldots, \Omega_{l}$, with $\Omega_{1}=\Omega(\mathbb{K})$ (where $l$ depends only on $G$ ). These translates can be chosen to be definable. For the elements $\gamma$ in $\Omega_{j}(\mathbb{K}) \backslash \bigcup_{i=0}^{j-1} \Omega_{i}(\mathbb{K})$, we define the ball $U_{\gamma, \mathbb{K}}$ in the same way, except using the coordinates on $\Omega_{j}$.

4.4.1. Completing the proof. Now we are ready to prove that there is a constructible motivic exponential function on $G_{0^{+}}^{\mathrm{reg}}$ whose specialization has the same restriction to $G(\mathbb{K})_{r}^{\text {reg }}$ as the function $\theta_{\pi}$. Let $r$ be the depth of $\pi$, as before (and recall that we are assuming that it is the essential depth of $\pi$ as well). Let $f_{\gamma}(x)$ be the characteristic function of $U_{\gamma, \mathbb{K}}$. Then by the local constancy result discussed above,

$$
\Theta_{\pi}\left(f_{\gamma}\right)=\operatorname{vol}\left(U_{\gamma, \mathbb{K}}\right) \theta_{\pi}(\gamma) .
$$

By Theorem $2.21(2,3)$, the functions $\frac{Q_{\mathfrak{g},-r}(x)}{\operatorname{deg}(x)} \Theta_{x}\left(f_{\gamma}\right)$ form a constructible family of constructible motivic exponential functions of $\gamma$, indexed by $x \in B_{\mathfrak{g},-r}$. Then, by Lemma 4.9 combined with (11), the functions

$$
\frac{Q_{\mathfrak{g},-r}(x)}{\operatorname{deg}(x)} \theta_{x}(\gamma)=q^{-n(\gamma)} \frac{Q_{\mathfrak{g},-r}(x)}{\operatorname{deg}(x)} \Theta_{x}\left(f_{\gamma}\right)
$$

form a constructible family of constructible motivic exponential functions, and we can define

$$
F(x, \gamma):=q^{-n(\gamma)} \frac{Q_{\mathfrak{g},-r}(x)}{\operatorname{deg}(x)} \Theta_{x}\left(f_{\gamma}\right),
$$


which completes the proof. We observe that dividing by the formal degree was only necessary to show that $F(x, \gamma)$ is constructible in the variable $x \in B_{\mathfrak{g},-r}$ (see Remark 2.24).

4.5. Proof of Theorem 2.23 in the positive characteristic case. As above, $\pi$ is a restricted representation of minimal depth $r$; see Section 2.6 .

Let us summarize the relevant results from the previous sections. Let $f_{a}$ be any constructible family of constructible motivic functions, with the parameter $a$ coming from some definable subassignment, and such that the support of $f_{a, \mathbb{K}}$ is contained in some fixed definable compact subset of $G(\mathbb{K})_{r}^{\text {reg }}$ for all $\mathbb{K} \in \mathcal{F}_{\mathbb{Z}, M_{1}}$ for some $M_{1}$. Then:

(1) There exist a definable subassignment $B_{\mathfrak{g},-r} \in \operatorname{RDef}_{\mathbb{Q}}$, and $M>0$, such that for every $\mathbb{K} \in \mathcal{F}_{\mathbb{Z}, M}$ and every restricted representation $\pi$ of $G(\mathbb{K})$ of minimal depth $r$, the values $\Theta_{\pi}\left(f_{a, \mathbb{K}}\right)$ depend only on $a$ and the image $x \in B_{\mathfrak{g}, r}\left(\mathbb{R}_{\mathbb{K}}\right)$ of $\pi$ under the map of Theorem 2.21(1), so we can write instead $\Theta_{x}\left(f_{a, \mathbb{K}}\right)$.

(2) $\Theta_{x}\left(f_{a}\right)$ is a constructible motivic exponential function of $a$. We denote it by $F_{x}(a)$.

Recall the family $\xi_{a, n}$ of definable test functions from Section 3.2.1, that spans a dense subspace of $C_{c}^{\infty}\left(G^{\mathrm{reg}}(\mathbb{K})\right)$ for all $\mathbb{K} \in \mathcal{F}_{\mathbb{Z}, M_{\xi}}$ for the constant $M_{\xi}$ that depends only on $G$. Let $M$ be the constant from the already proven characteristic zero case of Theorem 2.23 and let $M_{0}=\max \left(M, M_{\xi}\right)$. Then, for the fields of characteristic zero $\mathbb{K} \in \mathcal{A}_{\mathbb{Z}, M_{0}}$ in addition to the above two statements, we also have:

(3) There exists a constructible motivic exponential function $F$ on $B_{\mathfrak{g}, r} \times G^{\text {reg }}$ such that for all $\mathbb{K} \in \mathcal{A}_{\mathbb{Z}, M_{0}}$,

$$
F_{\mathbb{K}}(x, \gamma)=\frac{Q_{\mathfrak{g},-r, \mathbb{K}}(x)}{\operatorname{deg}(x)} \theta_{x}(\gamma) .
$$

(4) There exists a family of definable sets $U_{\gamma}$ (see Lemma 4.12), with $\gamma \in G^{\mathrm{reg}}$, such that for all fields $\mathbb{K} \in \mathcal{A}_{\mathbb{Z}, M_{0}}$, the character function $\theta_{x}$ is constant on $U_{\gamma, \mathbb{K}}$.

The lemma below asserts that we can transfer sufficient information about local constancy of the character to the positive characteristic case, using everything we already proved in the characteristic zero case.

Lemma 4.13. Let $\mathbb{K} \in \mathcal{B}_{\mathbb{Z}, M_{0}}$, and let $\pi$ be a restricted representation of $G(\mathbb{K})$ of minimal depth $r$. Then the function $\theta_{\pi}$ is constant on the sets $U_{\gamma, \mathbb{K}}$.

Proof. Let $x \in B_{\mathfrak{g},-r}\left(\mathbb{k}_{\mathbb{K}}\right)$ be the point that corresponds to the definable $r$-equivalence class of $\pi$. Let $\gamma_{0} \in G(\mathbb{K})_{r}^{\mathrm{reg}}$. Suppose that $g_{0} \in U_{\gamma_{0}, \mathbb{K}}$, and put $\theta_{\pi}\left(g_{0}\right)=b$. Then by local constancy, there is a neighbourhood $U_{b}$ of $g_{0}$ such that the character $\theta_{\pi}$ takes the value $b$ everywhere on $U_{b}$. We can assume that $U_{b} \subset U_{\gamma_{0}, \mathbb{K}}$. Let $\mathbf{1}_{U_{b}}$ be the characteristic function of the set $U_{b}$. Let $\left\{\eta_{a}\right\}_{a \in A}$ be any family of definable test functions satisfying the conditions (1) and (2) of Definition 2.20 for example, the family $\xi_{a, n}$ of Section 3.2.1. Then there is a definable compact set $W_{\mathbb{K}} \subset G(\mathbb{K})^{\text {reg }}$, containing $U_{b}$, and for every $\varepsilon>0$, a definable function $f_{\varepsilon}=\sum_{i=1}^{m} c_{i} \eta_{a_{i}, \mathbb{K}}$ (where the number $m$, the constants $c_{i} \in \mathbb{C}$, and the parameters $a_{i} \in A_{\mathbb{K}}$ depend on $\varepsilon$ ), such that $\operatorname{supp}\left(f_{\varepsilon}\right) \subset W_{\mathbb{K}}$, and

$$
\sup _{W_{\mathbb{K}}}\left|\mathbf{1}_{U_{b}}-f_{\varepsilon}\right|<\varepsilon .
$$


Let us evaluate the distribution character on the function $f_{\varepsilon}$. On the one hand, by definition of $\theta_{\pi}$,

$$
\begin{aligned}
\left|\Theta_{\pi}\left(f_{\varepsilon}\right)-b \operatorname{vol}\left(U_{b}\right)\right| & =\left|\int_{G(\mathbb{K})} \theta_{\pi}(g) f_{\varepsilon}(g) d g-\int_{G(\mathbb{K})} \theta_{\pi}(g) \mathbf{1}_{U_{b}}(g) d g\right| \\
& <\varepsilon \operatorname{vol}\left(W_{\mathbb{K}}\right) \sup _{W_{\mathbb{K}}}\left|\theta_{\pi}\right| .
\end{aligned}
$$

On the other hand, $\Theta_{\pi}\left(\eta_{a}\right)$ is a constructible motivic function of $a$, and it can be specialized both to the fields in $\mathcal{A}_{\mathbb{Z}, M_{0}}$ and in $\mathcal{B}_{\mathbb{Z}, M_{0}}$. By the characteristic-zero case of Theorem 2.23 (see 4 4.4), on $\mathcal{A}_{\mathbb{Z}, M_{0}}$, its specialization coincides with the specialization of the function

$$
\int_{G^{\mathrm{reg}}} \frac{\operatorname{deg}(x)}{Q_{\mathfrak{g},-r}(x)} F(x, g) \eta_{a}(g) d g
$$

of $a$, where $F$ is the function from condition (3) above.

Therefore, by the transfer principle (see e.g. [12, Theorem 2.7.2]), the specializations of these two functions to the fields in $\mathcal{B}_{\mathbb{Z}, M_{0}}$ also coincide. Hence, for every $i=0, \ldots, m$,

$$
\begin{aligned}
\Theta_{\pi}\left(\eta_{a_{i}, \mathbb{K}}\right) & =\left(\frac{\operatorname{deg}(x)}{Q_{\mathfrak{g},-r}(x)} \int_{G} F(x, g) \eta_{a}(g) d g\right)_{\mathbb{K}}\left(a_{i}\right) \\
& =\frac{\operatorname{deg}(x)}{Q_{\mathfrak{g},-r, \mathbb{K}}(x)} \int_{G(\mathbb{K})^{\mathrm{reg}}} F_{\mathbb{K}}(x, g) \eta_{a_{i}}(g) d g .
\end{aligned}
$$

Since, for all $\mathbb{K} \in \mathcal{A}_{\mathbb{Z}, M}$, the specialization of $F(x, g)$ to $\mathbb{K}$ is constant for $g \in U_{\gamma, \mathbb{K}}$, it also has to be constant for $\mathbb{K} \in \mathcal{B}_{\mathbb{Z}, M_{0}}$, and we have

$$
\int_{G(\mathbb{K})^{\mathrm{reg}}} F_{\mathbb{K}}(x, g) \mathbf{1}_{U_{b}}(g) d g=F_{\mathbb{K}}\left(x, \gamma_{0}\right) \operatorname{vol}\left(U_{b}\right) .
$$

We get

$$
\begin{array}{r}
\left|\Theta_{\pi}\left(f_{\varepsilon}\right)-\frac{\operatorname{deg}(x)}{Q_{\mathfrak{g},-r, \mathbb{K}}(x)} F_{\mathbb{K}}\left(x, \gamma_{0}\right) \operatorname{vol}\left(U_{b}\right)\right| \\
<\varepsilon \operatorname{vol}\left(W_{\mathbb{K}}\right) \sup _{g \in W_{\mathbb{K}}} F_{\mathbb{K}}(x, g) \frac{\operatorname{deg}(x)}{Q_{\mathfrak{g},-r, \mathbb{K}}(x)} .
\end{array}
$$

We observe that when the field $\mathbb{K}$ is fixed, and the point $x \in B_{\mathfrak{g},-r, \mathbb{K}}$ is fixed as well, while $\varepsilon$ and, accordingly, $a_{i}$ and the function $f_{\varepsilon}$ vary, all the factors in the right-hand side of the inequalities (14) and (12), except $\varepsilon$, remain constant. Letting $\varepsilon \rightarrow 0$ and comparing (14) with (12), we get

$$
\theta_{\pi}\left(g_{0}\right)=b=\frac{\operatorname{deg}(x)}{Q_{\mathfrak{g},-r, \mathbb{K}}(x)} F_{\mathbb{K}}\left(x, \gamma_{0}\right) .
$$

A similar argument shows that $\theta_{\pi}\left(\gamma_{0}\right)=\frac{\operatorname{deg}(x)}{Q_{\mathfrak{g},-r, \mathbb{K}}(x)} F_{\mathbb{K}}\left(x, \gamma_{0}\right)=b$, as desired.

Remark 4.14. A similar argument could be used to carry J. Korman's local constancy result of [31] over to the fields of positive characteristic, when the residue characteristic is large enough. On the other hand, this result in positive characteristic also follows from [5]. In general, the fact that the characters are motivic should have implications for their local constancy; this remains to be explored. 
Now we can prove Theorem 2.23 in full generality, not just for characteristic zero fields; we just need to repeat the conclusion of the proof of Theorem 2.23 from Section 4.4.1, which now works both in $\mathcal{A}_{\mathbb{Z}, M_{0}}$ and $\mathcal{B}_{\mathbb{Z}, M_{0}}$ by Lemma 4.13.

\section{Concluding Remarks And Future Questions}

5.1. Toward local integrability in positive characteristic. We believe that just using the information about the distribution character on our family of functions, one can prove the local integrability of the character function (at least in the range of validity of Theorem 2.21(2)). So far we have shown that, for all fields $\mathbb{K} \in \mathcal{F}_{\mathbb{Z}, M_{0}}$, every definable $r$-equivalence class of restricted representations of $G(\mathbb{K})$ of minimal depth $r$ corresponds to a point $x \in B_{\mathfrak{g},-r}\left(\mathbb{k}_{\mathbb{K}}\right)$, and the character $\theta_{\pi}$ on $G(\mathbb{K})_{r}^{r e g}$ is the restriction of the specialization of the constructible motivic exponential function $F(x, g)$, up to a rational constant $\frac{\operatorname{deg}(x)}{Q_{\mathfrak{g},-r, \mathbb{K}}(x)}$. Further, we know that for all fields $\mathbb{K} \in \mathcal{A}_{\mathbb{Z}, M_{0}}$ and $x \in B_{\mathfrak{g},-r}\left(\mathbb{k}_{\mathbb{K}}\right)$, the specialization of $F$ is locally integrable with respect to $g$. We expect that a general "transfer of integrability" result should hold for constructible motivic exponential functions; this will be explored in future work. Finally, once we have the local integrability result, we will be able to conclude that the distribution character is a continuous linear functional on the space $C_{c}^{\infty}\left(G(\mathbb{K})_{r}\right)$ for all $\mathbb{K} \in \mathcal{F}_{\mathbb{Z}, M}$, and therefore knowing its values on a definable family of definable test functions is sufficient.

5.2. Toward an explicit algorithm for computing character values. Suppose one wanted to write a computer program that took the residue field of characteristic $p$, and the rational number $r$ as an input, and produced a number $M$ and the character tables for representations of depth $r$ of $\operatorname{Sp}_{2 N}\left(\mathbb{Q}_{p}\right)$ and $\mathrm{SO}_{n}\left(\mathbb{Q}_{p}\right)$, for $p>M$ (here we take $\mathbb{Q}_{p}$ for simplicity so that we do not have to think of the implementation of the definition of a field extension; all of our arguments work for an arbitrary $p$-adic field). Here is what we can contribute so far toward such a program.

(1) The first step is to write a program that would generate the parameter space $B_{\mathfrak{g},-r}$ following the recipe given here and in [14. No integration is required.

(2) If motivic integration had been implemented, then one could further take a formula defining the test function $f$ as input, and for every such function with support contained in $G\left(\mathbb{Q}_{p}\right)_{r}^{\text {reg }}$, output the actual value of the distribution character evaluated at this function.

This already would make a lot of experimentation with characters possible. In particular, one could (near the identity) test the character for local constancy on specific sets, as long as those sets were definable; test various linear combinations of characters for stability, etc.

(3) Since we know that the character is a constructible function, we conclude that if an algorithm for cell decomposition had been implemented, we could produce, for each depth $r$, a complete list of neighbourhoods of regular elements of depth at least $r$ on which the function $\theta_{x}$ is constant, and the list of its values on those neighbourhoods. 
5.3. Future questions. There are a few things pleading to be carried out in the context of this paper, and we plan to address them in future work. First, there is folklore evidence that it should be possible to improve the local constancy result of [31, and our Theorem 2.23 corroborates that: the level sets of a constructible exponential function form a partition of its domain into definable subsets, and it seems that the sets $\gamma T(\mathbb{K})_{r^{\prime}+s(\gamma)}$ are not quite large enough to be definable because of the "extra" term $s(\gamma)$. We hope to obtain a rigorous proof along these lines.

Second, it is natural to investigate the character away from the identity. It is clear that the multiplicative characters of the field need to be included in the language in order to make similar treatment possible. We expect that, once this is done, it will be possible to describe the values of the Harish-Chandra character on the whole group.

\section{REFERENCES}

[1] Jeffrey D. Adler, Refined anisotropic K-types and supercuspidal representations, Pacific J. Math. 185 (1998), no. 1, 1-32. MR1653184(2000f:22019)

[2] Jeffrey D. Adler and Alan Roche, An intertwining result for $p$-adic groups, Canad. J. Math. 52 (2000), no. 3, 449-467. MR1758228 (2001m:22032)

[3] Jeffrey D. Adler and Stephen DeBacker, Some applications of Bruhat-Tits theory to harmonic analysis on the Lie algebra of a reductive $p$-adic group, with appendices by Reid Huntsinger and Gopal Prasad, Michigan Math. J. 50 (2002), no. 2, 263-286. MR 1914065 (2003g:22016)

[4] , Murnaghan-Kirillov theory for supercuspidal representations of tame general linear groups, J. Reine Angew. Math. 575 (2004), 1-35. MR2097545 (2005j:22008)

[5] Jeffrey D. Adler and Jonathan Korman, The local character expansion near a tame, semisimple element, Amer. J. Math. 129 (2007), no. 2, 381-403. MR.2306039 (2008a:22020)

[6] Jeffrey D. Adler and Loren Spice, Supercuspidal characters of reductive p-adic groups, Amer. J. Math 131, no. 4, 1137-1210. MR2543925 (2011a:22018)

[7] François Bruhat and Jacques Tits, Groupes réductifs sur un corps local, Inst. Hautes Études Sci. Publ. Math. 41 (1972), 5-251 (French). MR0327923 (48 \#6265)

[8] _ Groupes réductifs sur un corps local. II. Schémas en groupes. Existence d'une donnée radicielle valuée, Inst. Hautes Études Sci. Publ. Math. 60 (1984), 197-376 (French). MR:756316 (86c:20042)

[9] Raf Cluckers and François Loeser, Ax-Kochen-Eršov theorems for p-adic integrals and motivic integration, Geometric methods in algebra and number theory, Progr. Math., vol. 235, Birkhäuser Boston, Boston, MA, 2005, pp. 109-137. MR2159379 (2006g:12014)

[10] Raf Cluckers and François Loeser, Constructible exponential functions, motivic Fourier transform and transfer principle, Annals of Mathematics 171 (2010), no. 2, 1011-1065. MR:2630060

[11] Constructible motivic functions and motivic integration, Invent. Math. 173 (2008), no. 1, 23-121. MR.2403394

[12] Raf Cluckers, Thomas Hales, and François Loeser, Transfer principle for the Fundamental Lemma, available at arXiv:0712.0708. To appear.

[13] Lawrence Corwin, Allen Moy, and Paul J. Sally Jr., Supercuspidal character formulas for $\mathrm{GL}_{\ell}$, Representation theory and harmonic analysis (Cincinnati, OH, 1994), 1995, pp. 1-11. MR:1365530 (96m:22037)

[14] Clifton Cunningham and Thomas C. Hales, Good orbital integrals, Represent. Theory 8 (2004), 414-457 (electronic). MR2084489 (2006d:22021)

[15] Stephen DeBacker and Mark Reeder, Depth-zero supercuspidal L-packets and their stability, Ann. of Math. 169 (2009), no. 3, 795-901. MR2480618 (2010d:22023)

[16] Julia Gordon and Thomas C. Hales, Virtual transfer factors, Represent. Theory 7 (2003), 81-100 (electronic). MR 1973368(2004a:11084)

[17] Julia Gordon, Motivic nature of character values of depth-zero representations, Int. Math. Res. Not. 34 (2004), 1735-1760. MR2057872 (2005c:22026) 
[18] _ Motivic Haar measure on reductive groups, Canad. J. Math. 58 (2006), no. 1, 93114. MR2195593 (2006m:14059)

[19] Julia Gordon and Yoav Yaffe, An Overview of Arithmetic Motivic Integration, Ottawa lectures on Admissible Representations of reductive $p$-adic groups, Fields Institute Monograph series, vol. 26, American Mathematical Society, Providence, RI; Fields Institute for Research in Mathematical Sciences, Toronto, ON, 2009. MR2508723 (2010i:14020)

[20] Jeffrey Hakim and Fiona Murnaghan, Distinguished tame supercuspidal representations, IMRP 2 (2008). MR2431732 (2010a:22022)

[21] Thomas C. Hales, Can p-adic integrals be computed?, Contributions to automorphic forms, geometry, and number theory, Johns Hopkins University Press, Baltimore, MD, 2004, pp. 313-329. MR.2058612(2005d:11168)

[22] What is motivic measure?, Bull. Amer. Math. Soc. (N.S.) 42 (2005), no. 2, 119-135. MR.213307 (2006h:14031)

[23] - Hyperelliptic curves and harmonic analysis (why harmonic analysis on reductive p-adic groups is not elementary), Representation theory and analysis on homogeneous spaces (Simon Gindikin, Frederick P. Greenleaf, and Paul J. Sally Jr., eds.), Contemporary Mathematics, vol. 177, American Mathematical Society, Providence, RI, 1994, pp. 137-169. MR:1303604 (96d:22024)

[24] Harish-Chandra, Harmonic analysis on reductive p-adic groups, notes by G. van Dijk, Springer-Verlag, Berlin, 1970. MR0414797 (54 \#2889)

[25] Ehud Hrushovski and David Kazhdan, Integration in valued fields, Progr. Math., vol. 253, Birkhäuser Boston, Boston, MA, 2006. MR2263194 (2007k:03094)

[26] Noriaki Kawanaka, Shintani lifting and Gel'fand-Graev representations, The Arcata Conference on Representations of Finite Groups (Arcata, Calif., 1986), 1987, pp. 147-163. MR:933357 (89h:22037)

[27] David Kazhdan and George Lusztig, Fixed point varieties on affine flag manifolds, Israel J. Math. 62 (1988), no. 2, 129-168. MR947819 (89m:14025)

[28] Ju-Lee Kim and Fiona Murnaghan, K-types and $\Gamma$-asymptotic expansions, J. Reine Angew. Math. 592 (2006), 189-236. MR 2222734 (2007m:22016)

[29] Ju-Lee Kim, Supercuspidal representations: an exhaustion theorem, J. Amer. Math. Soc. 20 (2007), no. 2, 273-320 (electronic). MR.2276772

[30] Ju-Lee Kim and Allen Moy, Involutions, classical groups, and buildings, J. Algebra 242 (2001), no. 2, 495-515. MR1848956 (2003d:51009)

[31] Jonathan Korman, On the local constancy of characters, J. Lie Theory 15 (2005), no. 2, 561-573. MR2147443

[32] Robert E. Kottwitz, Transfer factors for Lie algebras, Represent. Theory 3 (1999), 127-138 (electronic). MR:1703328 (2000g:22028)

[33] Allen Moy and Gopal Prasad, Unrefined minimal K-types for p-adic groups, Invent. Math. 116 (1994), no. 1-3, 393-408. MR1253198 (95f:22023)

[34] _ Jacquet functors and unrefined minimal K-types, Comment. Math. Helv. 71 (1996), no. 1, 98-121. MR.1371680 (97c:22021)

[35] Fiona Murnaghan, Characters of supercuspidal representations of classical groups, Ann. Sci. École Norm. Sup. (4) 29 (1996), no. 1, 49-105. MR1368705 (98c:22016)

[36] Mark Reeder, Supercuspidal L-packets of positive depth and twisted Coxeter elements, J. Reine Angew. Math. 620 (2008), 1-33. MR2427973 (2009e:22019)

[37] François Rodier, Modèle de Whittaker et caractères de représentations, Non-commutative harmonic analysis (J. Carmona, J. Dixmier, and M. Verne, eds.), Lecture Notes in Mathematics, vol. 466, Springer-Verlag, Berlin, 1975, pp. 151-171. MR0393355(52 \#14165)

[38] Tetsuya Takahashi, Formulas for tamely ramified supercuspidal characters of GL3, Trans. Amer. Math. Soc. 355 (2003), no. 2, 567-591 (electronic). MR 1932714 (2003i:22020)

[39] - On some constants in the supercuspidal characters of $\mathrm{GL}_{l}, l$ a prime $\neq p$, Trans. Amer. Math. Soc. 357 (2005), no. 6, 2509-2526 (electronic). MR2140448 (2006d:22027)

[40] Jean-Loup Waldspurger, Intégrales orbitales nilpotentes et endoscopie pour les groupes classiques non ramifiés, Astérisque 269 (2001), vi+449. MR.1817880 (2002h:22014:22018)

[41] Yimu Yin, Special transformations in algebraically closed valued fields, Ann. Pure Appl. Logic 161 (2010), no. 12, 1541-1564. MR2674050

[42] — Grothendieck homomorphisms in algebraically closed valued fields III: Fourier transform, available at arXiv:0903.1097. preprint. 
[43] - Integration in algebraically closed valued fields, Ann. Pure Appl. Logic 162 (2011), no. 5, 384-408.

[44] Jiu-Kang Yu, Construction of tame supercuspidal representations, J. Amer. Math. Soc. 14 (2001), no. 3, 579-622 (electronic). MR,1824988 (2002f:22033)

Université Lille 1, Laboratoire Painlevé, CNRS - UMR 8524, Cité Scientifique, 59655 Villeneuve d'Ascq Cedex, France, and, Katholieke Universiteit Leuven, Department of Mathematics, Celestijnenlaan 200B, B-3001 Leuven, Belgium

E-mail address: Raf.Cluckers@math.univ-lille1.fr

URL: http://math.univ-lille1.fr/ cluckers

Department of Mathematics, University of Calgary

E-mail address: cunning@math.ucalgary.ca

Department of Mathematics, University of British Columbia

E-mail address: gor@math.ubc.ca

Department of Mathematics, Texas Christian University

E-mail address: 1.spice@tcu.edu 